\title{
The plastikstufe - a generalization of the overtwisted disk to higher dimensions
}

\author{
KLAUS NIEDERKRÜGER
}

\begin{abstract}
In this article, we give a first prototype-definition of overtwistedness in higher dimensions. According to this definition, a contact manifold is called overtwisted if it contains a plastikstufe, a submanifold foliated by the contact structure in a certain way. In three dimensions the definition of the plastikstufe is identical to the one of the overtwisted disk. The main justification for this definition lies in the fact that the existence of a plastikstufe implies that the contact manifold does not have a (semipositive) symplectic filling.
\end{abstract}

53D10, 57R17; 53D35

The situation of contact topology can be roughly stated like this: the 3-dimensional contact manifolds can be understood very adequately by topological methods, a farreaching classification has been achieved and relations to many other fields have been established. In contrast, the world map of higher-dimensional contact geometry consists almost entirely of white spots. A powerful method for constructing such manifolds is contact surgery, the most promising technique developed so far to distinguish different contact structures is contact homology and with Giroux's open book decomposition, it is hoped that some classification results could be obtained.

The first structural distinction found for contact 3-manifolds was the notion of overtwistedness. It turned out that such manifolds firstly do not allow an (even weak) symplectic filling by Eliashberg [4] and Gromov [8], and secondly can be classified in a very satisfactory way as in Eliashberg [3].

In higher dimensions, surprisingly, no analogous criterion has yet been found. Giroux has proposed a definition based on his open book decomposition, which in three dimensions is completely equivalent to the standard one. In contrast, our definition is based on the existence of a plastikstufe, a direct generalization of the overtwisted disk. In Gromov's famous paper on holomorphic curves [8], a sketchy description of something, which possibly could be a plastikstufe, is given. The generalization of overtwistedness described in this article was found independently by Yuri Chekanov. Interestingly, his (unpublished) proof of Theorem 1 uses very different methods. 
The definition of the plastikstufe given in this paper is certainly only a preliminary version, meant as a prototype leading to a criterion for nonfillability in higher dimensions. Our definition implies the following theorem.

Theorem 1 Let $(M, \alpha)$ be a contact manifold containing an embedded plastikstufe. Then $M$ does not have any semipositive symplectic filling. If $\operatorname{dim} M \leq 5$, then $M$ does not have any symplectic filling at all.

The proof of this statement will be given in Section 2 .

Remark 1 A $2 n$-dimensional symplectic manifold $(M, \omega)$ is called semipositive if every $A \in \pi_{2}(M)$ with $\omega(A)>0$ and $c_{1}(A) \geq 3-n$ has nonnegative Chern number. Note that every symplectic $4-$ or 6 -manifold is semipositive.

There are several shortcomings of the definition of overtwisted given here, the most important being that no example of a closed contact manifold containing an embedded plastikstufe has been found so far. It is relatively easy though to construct open manifolds containing an embedded plastikstufe. As observed by Chekanov, the plastikstufe can be used to detect exotic contact structures on $\mathbb{R}^{2 n-1}$ (see Section 5.1).

From a practical viewpoint the definition of the plastikstufe is also rather cumbersome, because it is less topological than the overtwisted disk in dimension 3 (Remark 5).

Remark 2 At the time of the final revision of this article, Francisco Presas Mata announced a method which allows one to construct closed contact manifolds that contain embedded plastikstufes [12].

Acknowledgments The work on this article was initiated at the Universität zu Köln and finished at the Université Libre de Bruxelles. My research was funded in Germany by the university and in Belgium by the Fonds National de la Recherche Scientifique (FNRS).

The definition for the plastikstufe given in this article was found after innumerable discussions with Kai Zehmisch. Later, it was Frédéric Bourgeois who guided me in converting the intuitive picture I had in mind into sound and hard mathematics. I spoke with Otto van Koert during the whole project almost on a daily basis. These conversations helped me find solutions to many problems in this article. Without these three colleagues, this article wouldn't have been possible. Furthermore, Francisco Presas Mata helped me solve the last steps in the proof. I also profited from discussions with Yuri Chekanov, Hansjörg Geiges, Ferit Öztürk, Dietmar Salamon, Felix Schlenk and Savaş Yazıcı. 


\section{Preliminaries}

The following notions are standard in symplectic topology, but for completeness we briefly repeat them here.

Definition Let $(W, \omega)$ be a symplectic manifold. A Liouville vector field $X_{L}$ is a vector field on $W$, whose flow makes the symplectic form expand exponentially. This property can be formulated equivalently as

$$
\mathcal{L}_{X_{L}} \omega=\omega
$$

A (convex) symplectic filling $(W, \omega)$ of a contact manifold $(M, \alpha)$ is a compact symplectic manifold with boundary $\partial W=M$, such that there exists a Liouville vector field $X_{L}$ in a neighborhood of $\partial W$ that points outwards, and such that

$$
\alpha=\left.\left(\iota_{X_{L}} \omega\right)\right|_{T M} .
$$

Remark 3 We can define a function $h: U \rightarrow(-\infty, 0]$ on a neighborhood $U \subset W$ of $M$, by considering the time $t_{p}$ it takes a point $p \in U$ to flow along $X_{L}$ to $M$, and then setting $h(p):=-t_{p}$. Define $\tilde{\alpha}:=\iota_{X_{L}} \omega$. By taking on each level set $M_{t}:=h^{-1}(t)$ (sufficiently close to $M$ ) the Reeb field of the contact form $\alpha_{t}:=\left.\widetilde{\alpha}\right|_{T M_{t}}$, we obtain a smooth vector field, which we denote by $X_{\text {Reeb }}$.

In the context of this article we will use the term "compatible almost complex structure" in the following sense.

Definition Let $(W, \omega)$ be a symplectic filling of a contact manifold $(M, \alpha)$. A compatible almost complex structure $J$ is a smooth section of the endomorphism bundle $\operatorname{End}(T W)$ such that $J^{2}=-\mathbb{1}$, that is compatible with $\omega$ in the usual sense, which means that for all $X, Y \in T_{p} W$, the following equation holds

and

$$
\omega(J X, J Y)=\omega(X, Y),
$$

$$
g(X, Y):=\omega(J X, Y)
$$

defines a Riemannian metric. Additionally, we require $J$ to satisfy close to the boundary $M=\partial W$ the following properties: for the two vector fields $X_{L}$ and $X_{\text {Reeb }}$ introduced above, $J$ is defined as

$$
J X_{L}=X_{\text {Reeb }} \quad \text { and } \quad J X_{\text {Reeb }}=-X_{L},
$$

and $J$ leaves the subbundle $\xi_{t}=\operatorname{ker} \alpha_{t} \leq T M_{t}$ invariant. 
Proposition 2 Let $u: V \cap \mathbb{U} \rightarrow W$ be a $J$-holomorphic map $(V \subset \mathbb{C}$ is an open set, and $\mathbb{M} \subset \mathbb{C}$ is the upper halfplane). The function $h \circ u: V \cap \mathbb{H} \rightarrow \mathbb{R}$ is subharmonic.

Proof A short computation shows that

$$
\begin{aligned}
0 & \leq u^{*} \omega=u^{*} d \iota_{X_{L}} \omega=u^{*} d \tilde{\alpha}=u^{*} d(-d h \circ J)=-u^{*} d d^{c} h \\
& =-d d^{c}(h \circ u)=\left(\frac{\partial^{2} h \circ u}{\partial x^{2}}+\frac{\partial^{2} h \circ u}{\partial y^{2}}\right) d x \wedge d y .
\end{aligned}
$$

Corollary 3 By the strong maximum principle and the boundary point lemma (eg Gilbarg and Trudinger [6]), any $J$-holomorphic curve $u:(\Sigma, \partial \Sigma) \rightarrow(W, \partial W)$ is either constant or it intersects $M=\partial W$ only at $\partial \Sigma$, and this intersection is transverse.

Finally, we will denote the moduli space of $J$-holomorphic disks lying in $U$ with boundary in $\tilde{U}$ and with one marked point $z_{0} \in \partial \mathbb{D}^{2}$ by the symbol $\mathcal{M}\left(U, \widetilde{U}, z_{0}\right)$.

\section{Definition of the plastikstufe}

Before giving the definition of the plastikstufe, we first introduce some preliminary definitions.

Definition A maximally foliated submanifold $L$ in a $(2 n-1)$-dimensional contact manifold $(M, \alpha)$ is a submanifold of dimension $n$ on which ker $\left.\alpha\right|_{T L}$ defines a (possibly singular) foliation.

Remark 4 The term "maximally" in the definition above means that $L$ is not contained in some higher dimensional submanifold also foliated by $\alpha$. The condition on the dimension is imposed by the fact that the leaves of the foliation are locally Legendrian submanifolds.

Remark 5 Frobenius' Theorem implies that $L$ is foliated by $\left.\alpha\right|_{T L}$ if and only if $\left.(\alpha \wedge d \alpha)\right|_{T L} \equiv 0$.

Remark 6 The main reason why these submanifolds are interesting in the setting of this paper, is that if $(M, \alpha)$ is the convex boundary of a symplectic manifold $(W, \omega)$, and $W$ is given an almost complex structure $J$ compatible on $M$ with $\xi=\operatorname{ker} \alpha$, then a maximally foliated submanifold $L \hookrightarrow M$ will be (at the nonsingular points of the foliation) a totally real submanifold in $W$ such that the Fredholm theory of $J$-holomorphic curves can be applied. 
Definition An elliptic singular set $S$ inside a maximally foliated submanifold $L$ is a closed codimension 2 submanifold inside $L$ whose neighborhood is diffeomorphic to $\mathbb{D}^{2} \times S \hookrightarrow L$ with coordinates $(x, y ; s)$ such that $\left.\alpha\right|_{T L}$ is represented by $x d y-y d x$ on this neighborhood (see Figure 1).

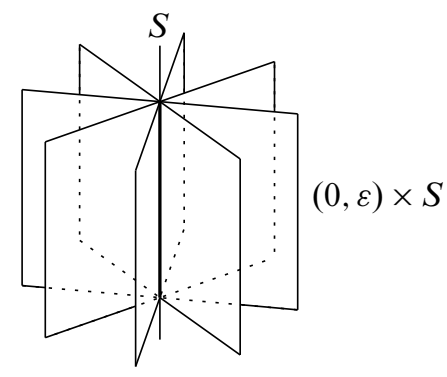

Figure 1: The foliation around a set of elliptic singularities consists of a circle of stripes $(0, \varepsilon) \times S$ forming a circle of rays touching in the singular set.

Example 1 It is very easy to find examples of maximally foliated submanifolds with elliptic singularities (at least locally). As Figure 1 already suggests, there is a similarity between an elliptic singularity and the binding of an open book.

By a result of Giroux [7], every contact manifold $(M, \alpha)$ has a compatible open book decomposition $(P, \vartheta)$ with binding $\left(B, \alpha_{B}\right)$, where $B=\partial P$ and $\alpha_{B}=\left.\alpha\right|_{T B}$. The normal form of the neighborhood of $\left(B, \alpha_{B}\right)$ can then be chosen to be

$$
\left(B \times \mathbb{D}^{2}, \alpha_{B}+\frac{1}{2}(x d y-y d x)\right),
$$

where $(x, y)$ are the coordinates on the 2-disk. If one chooses any Legendrian submanifold $S$ inside $B$ (which exist in abundance by Ekholm, Etnyre and Sullivan [2]), then the set $S \times \mathbb{D}^{2} \hookrightarrow B \times \mathbb{D}^{2}$ is a maximally foliated submanifold with elliptic singular set $S$.

An interesting problem would then be to try extend this submanifold into $M-B$.

The following definition is fundamental in 3-dimensional contact topology.

Definition Let $(M, \alpha)$ be a 3-dimensional contact manifold. An embedded 2-disk

$$
\iota: \mathbb{D}^{2} \hookrightarrow M
$$

is called an overtwisted disk $\mathbb{D}_{\mathrm{OT}}$, if there is only one point on the disk where the foliation given by $\iota^{*} \alpha$ is singular, and if the boundary of $\mathbb{D}_{\mathrm{OT}}$ is the only closed leaf of this foliation (see Figure 2).

Algebraic ${ }^{6} \mathcal{G}$ Geometric Topology, Volume 6 (2006) 


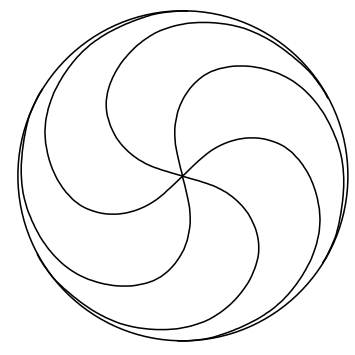

Figure 2: Foliation induced by $\iota^{*} \alpha$ on the overtwisted disk

Now we will give a conceivable generalization to higher dimensions: let $(M, \alpha)$ be a $(2 n-1)$-dimensional contact manifold, and let $S$ be a closed $(n-2)$-dimensional manifold.

Definition A plastikstufe $\mathcal{P} \mathcal{S}(S)$ with singular set $S$ in $M$ is an embedding of the n-dimensional manifold

$$
\iota: \mathbb{D}^{2} \times S \hookrightarrow M
$$

that is maximally foliated by the $1-$ form $\beta:=\iota^{*} \alpha$. The boundary $\partial \mathcal{P S}(S)$ of the plastikstufe should be the only closed leaf, and there should be an elliptic singular set at $\{0\} \times S$. The rest of the plastikstufe should be foliated by an $\mathbb{S}^{1}$-family of stripes, each one diffeomorphic to $(0,1) \times S$, which are spanned between the singular set on one end and approach $\partial \mathcal{P} \mathcal{S}(S)$ on the other side asymptotically.

Remark 7 An overtwisted disk $\mathbb{D}_{\mathrm{OT}}$ is equal to a 2-dimensional plastikstufe $\mathcal{P} \mathcal{S}(\{p\})$.

Remark 8 As mentioned in Remark 5 , $\operatorname{ker} \beta$ defines a foliation on $\mathbb{D}^{2} \times S$, if and only if $\beta \wedge d \beta \equiv 0$. This means that the definition above requires $\iota$ to satisfy a partial differential equation, in contrast to 3 -dimensional contact topology, where the foliation condition is trivially satisfied.

Remark 9 The boundary of the plastikstufe is a Legendrian submanifold $\partial \mathcal{P} \mathcal{S}(S) \cong$ $\mathbb{S}^{1} \times S$ of $M$.

Definition A $(2 n-1)$-dimensional contact manifold $(M, \alpha)$ will be called overtwisted if it contains an $n$-dimensional plastikstufe. 


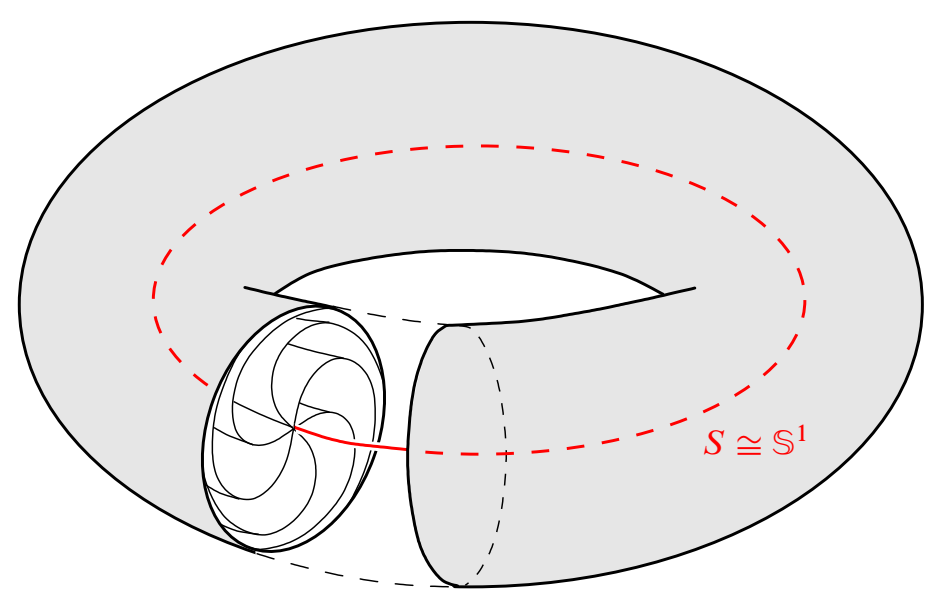

Figure 3: The plastikstufe in a 5-dimensional contact manifold

Example 2 It is easy to construct for any closed manifold $S$ an (unfortunately only) open contact manifold that contains the plastikstufe $\mathcal{P} \mathcal{S}(S)$ : let $\left(N^{3}, \alpha_{0}\right)$ be an overtwisted 3-manifold with overtwisted disk $\iota_{0}: \mathbb{D}_{\mathrm{OT}} \hookrightarrow N$. Let $M$ be the $(2 n-1)$-dimensional manifold $M:=N \times T^{*} S$ with the 1 -form

$$
\alpha=\alpha_{0}+\lambda_{\text {can }},
$$

where $\lambda_{\text {can }}=-\mathbf{p} \cdot d \mathbf{q}$ is the canonical $1-$ form of the cotangent bundle $T^{*} S$.

The 1 -form $\alpha$ is a contact form, and it is easy to check that the embedding

$$
\iota: \mathcal{P S}(S) \hookrightarrow M,(p, s) \mapsto\left(\iota_{0}(p), \sigma_{0}(s)\right),
$$

where $\sigma_{0}: S \hookrightarrow T^{*} S$ is the zero-section, really defines an $n$-dimensional plastikstufe.

\section{Sketch of the proof of Theorem 1}

The proof follows the line of the original proof in dimension 3 . We study a certain moduli space $\mathcal{M}$ of holomorphic disks, and there we find a cycle representing the trivial homology class in $\mathcal{M}$, but at the same time we show that it is mapped by the evaluation map to a nontrivial element of homology thus producing a contradiction.

Let $(M, \alpha)$ be a $(2 n-1)$-dimensional closed compact manifold that contains a plastikstufe $\mathcal{P} \mathcal{S}(S)$. Assume that $(W, \omega)$ is a semipositive symplectic filling of $M$. We will choose a compatible almost complex structure $J$ on $W$ (in the sense of the definition 
given in Section 0). The most important properties of $J$-holomorphic curves with respect to the convex boundary $M$ is that a holomorphic curve $u: \Sigma \rightarrow W$ cannot be tangent to $M$, and in particular if $p \in u(\Sigma)$ is a point, where the holomorphic curve intersects $M$, then $T_{p} u \cap \xi_{p}=\{0\}$. Note that $T_{p} u=0$ at a point $p \in M$ implies that $u \equiv p$.

In the rest of the proof, we consider the $J$-holomorphic disks $u: \mathbb{D}^{2} \rightarrow W$, whose boundary $u\left(\partial \mathbb{D}^{2}\right)$ lies on the plastikstufe, and which have a marked point $z_{0} \in \partial \mathbb{D}^{2}$ on their boundary. If $u \neq \equiv$ const, then $u\left(\partial \mathbb{D}^{2}\right)$ is transverse to the foliation on $\mathcal{P S}(S)$, and $u\left(\partial \mathbb{D}^{2}\right)$ cannot touch the boundary of $\mathcal{P S}(S)$. The boundary of every such curve is linked with the singular set $S$, because otherwise it could not be transverse to the foliation. This also implies that the only constant curves in the compactification of the moduli space lie in $S$.

In Section 3, we study the Bishop family of disks emanating from the singular set of the plastikstufe: for a small neighborhood $U$ of $S$, we find a standard model, where we can choose a carefully prepared almost complex structure $J$ (which can be extended to a regular $J$ on the whole symplectic filling). For this $J$ and for $\widetilde{U}:=U \cap \mathcal{P S}(S)$, the evaluation map $\operatorname{ev}_{z_{0}}$ is a diffeomorphism between the moduli space $\mathcal{M}\left(U, \tilde{U}, z_{0}\right)$ and $\tilde{U}$. Stated differently, for every point $p \in \widetilde{U}$ there is a unique holomorphic disk $u$ lying inside $U$ such that $u\left(z_{0}\right)=p$. We can explicitly write down this continuous family

$$
\psi: \widetilde{U} \rightarrow \mathcal{M}\left(W, \mathcal{P S}(S), z_{0}\right)
$$

such that $\operatorname{ev}_{z_{0}} \circ \psi=\mathrm{id}_{\tilde{U}}$. The curves $\psi(s)$ for every $s \in S$ are constant disks, and the boundary of every other disk $\psi(p)$ with $p \notin S$ is linked once with the singular set $S$.

Next, we show that the model neighborhood $U$ is foliated by compact codimension 2 manifolds, which are $J$-holomorphic. An intersection argument then allows us to show that any holomorphic disk $u$ lying partially in $U$ and whose boundary can be capped off by attaching a disk lying inside $\mathcal{P} \mathcal{S}(S)$ has to be completely contained in $U$. Hence the disk $u$ lies in the image of the map $\psi$.

With the results obtained in Section 3, one can see that the $N$-dimensional moduli space of holomorphic disks $(N=\operatorname{dim} \mathcal{P S}(S))$ described above only has a single end touching the singular set $S$, and stays otherwise at a finite distance from $S$.

The compactness result of Gromov states that the moduli space of holomorphic disks with bounded energy is always compact, provided we allow bubbling. More precisely, in a compact symplectic manifold every moduli space of simple disks with uniformly bounded energy, whose boundary sits on a compact totally real submanifold is a smooth manifold that can be compactified by including bubbled curves. In Section 4, we first 


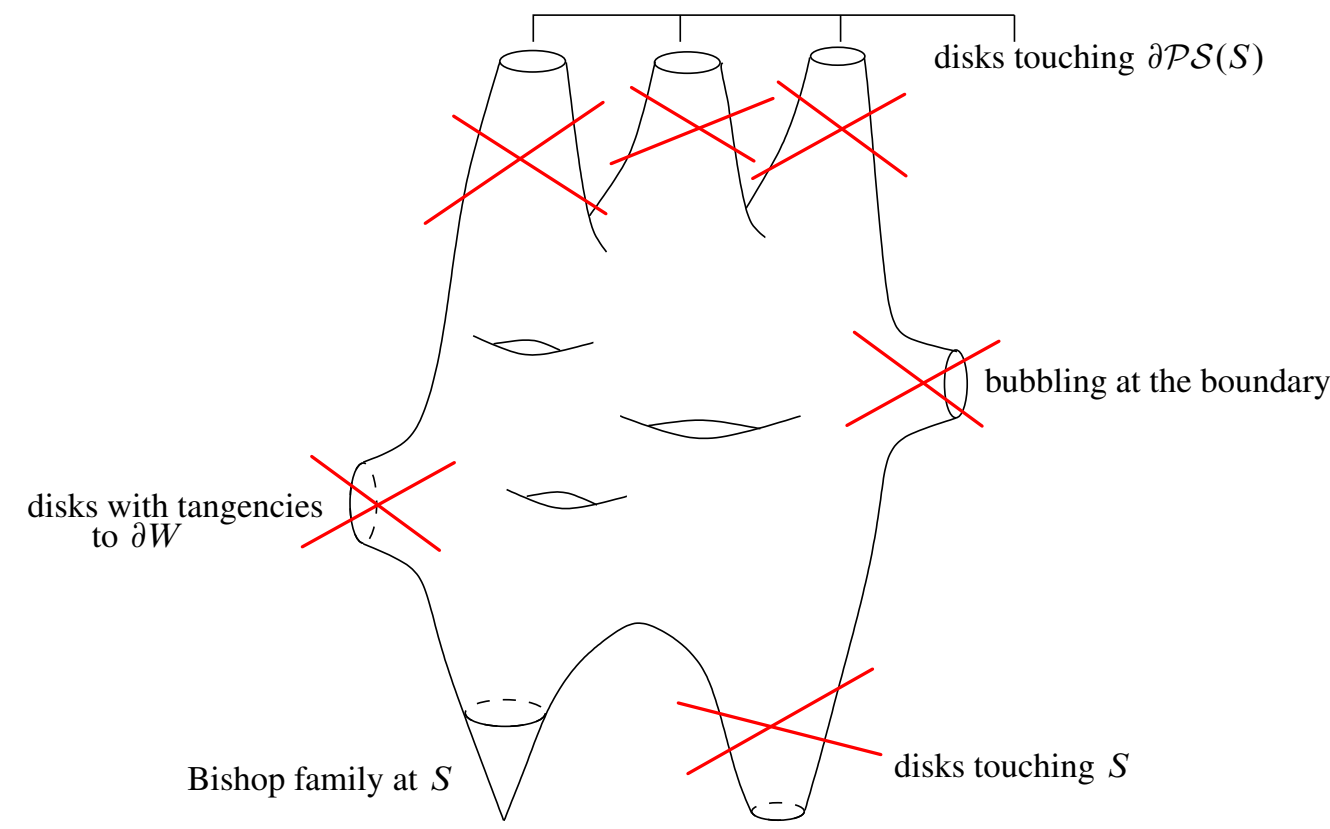

Figure 4: The possible boundary components of the moduli space $\mathcal{M}\left(W, \mathcal{P S}(S), z_{0}\right)$ are given by disks touching the singular set $S$ or the boundary of the plastikstufe $\partial \mathcal{P} \mathcal{S}(S)$, disks with tangencies to the boundary of the symplectic manifold $\partial W$, or curves having bubbles at their boundary. Different arguments show that with exception of the Bishop family all of these cases can be excluded.

show that there is a uniform energy bound for all curves in $\mathcal{M}\left(W, \mathcal{P} S(S), z_{0}\right)$. In our situation, $\mathcal{P S}(S)-S$ is not a compact totally real manifold, but since only the Bishop family comes close to $S$, the compactness property still holds in a suitable sense. There are two possible types of bubbles that can occur: either a sphere can bubble off at the interior of the holomorphic disks or a new holomorphic disk can form at the boundary of the disks. The foliation of the plastikstufe $\mathcal{P} \mathcal{S}(S)$ imposes a constraint on holomorphic curves, which forbids the second type of bubbling.

The treatment of interior bubbles is more technical, so we will first assume that no bubbling at all can happen to illustrate more easily the geometrical idea of the proof. The moduli space $\mathcal{M}\left(W, \mathcal{P} \mathcal{S}(S), z_{0}\right)$ is a smooth manifold, which could have several "boundary components" (see Figure 4). By the arguments given so far (and by the assumption that no spheres can bubble off), the only boundary of the moduli space corresponds to the Bishop family $\psi$ (see Figure 5). 
With the evaluation map at $z_{0} \in \partial \mathbb{D}^{2}$, it is easy to see that

$$
C_{\varepsilon}:=\{\psi(p) \mid p \in \mathcal{P S}(S), d(p, S)=\varepsilon\},
$$

for $\varepsilon>0$ sufficiently small, maps to the generator of $H_{N-1}(\mathcal{P S}(S)-S)$ with $N=$ $\operatorname{dim} \mathcal{P S}(S)$. Hence it follows that $C_{\varepsilon}$ has to represents a nontrivial cycle in the moduli space (see Figure 5). But this leads to a contradiction, because $C_{\varepsilon}$ is the only boundary component of the moduli space (if we remove all $C_{r}$ with $r<\varepsilon$ ), and hence it represents a boundary in homology.

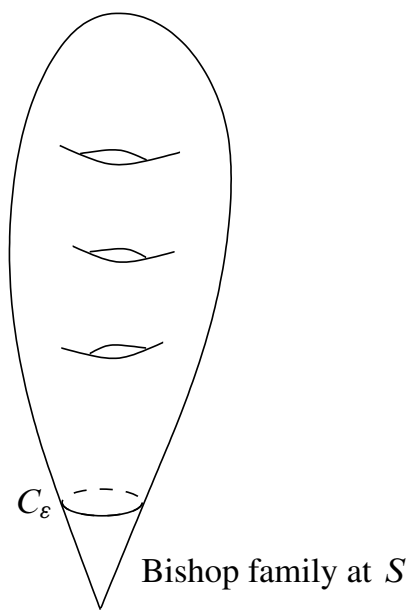

Figure 5: The moduli space $\mathcal{M}\left(W, \mathcal{P S}(S), z_{0}\right)$ only has one boundary component, and hence it follows that $\left[C_{\varepsilon}\right]$ represent the trivial class in $H_{N}\left(\mathcal{M}\left(W, \mathcal{P S}(S), z_{0}\right)\right)$ for $N=\operatorname{dim} \mathcal{M}-1$.

The conclusion is that the Bishop family cannot be the only boundary component of the moduli space. The only assumption made to exclude other components was that $W$ is a convex filling of the contact manifold $M$. This assumption must be false, and hence $M$ is not fillable.

The general situation, ie the one where interior bubbles can occur, can be treated like this: trace a path $\gamma(I)$ (with $I=[0,1]$ ) on $\mathcal{P} \mathcal{S}(S)$ running from the singular set $S$ to the boundary $\partial \mathcal{P S}(S)$. If the evaluation map ev $z_{z_{0}}: \mathcal{M}\left(W, \mathcal{P S}(S), z_{0}\right) \rightarrow \mathcal{P S}(S)$ is transverse to $\gamma$, then $\operatorname{ev}_{z_{0}}^{-1}(\gamma(I))$ will be a smooth 1-dimensional submanifold of 
$\mathcal{M}\left(W, \mathcal{P} \mathcal{S}(S), z_{0}\right)$. Define a projection $\pi$ by

$$
\begin{gathered}
\pi: \mathcal{P S}(S)-S \rightarrow \partial \mathcal{P} \mathcal{S}(S) \\
(z, s) \mapsto\left(\frac{z}{|z|}, s\right) .
\end{gathered}
$$

The preimage of a point $\left(e^{i \varphi_{0}}, s_{0}\right)$ under $\pi$ is a radial line in the plastikstufe joining $S$ and $\partial \mathcal{P S}(S)$. By Sard's Theorem, the set of singular values of the map $\pi \circ \mathrm{ev}_{z_{0}}$ has measure 0 , and because that map is surjective (consider the restriction to the Bishop family $\psi$ ), we find plenty of radial curves $\gamma$ such that $\mathrm{ev}_{z_{0}}^{-1}(\gamma(I))$ will be a 1-dimensional submanifold of $\mathcal{M}\left(W, \mathcal{P S}(S), z_{0}\right)$.

In Section 4, we show that the evaluation map $\mathrm{ev}_{z_{0}}$ is a pseudocycle (in the sense of McDuff and Salamon [10]), which means that the image of the evaluation map on the complement $\overline{\mathcal{M}}-\mathcal{M}$ (ie on the bubbled curves) lies in the closed image of finitely many codimension 2 manifolds. The projection of this set under $\pi$ still has at least codimension 1 , and hence, almost every point in $\partial \mathcal{P} \mathcal{S}(S)$ is a regular value of $\pi \circ \mathrm{ev}_{z_{0}}$ and not contained in the image of the bubbled curves.

For such a value $\left(e^{i \varphi_{0}}, s_{0}\right)$, the corresponding preimage in $\mathcal{M}\left(W, \mathcal{P} \mathcal{S}(S), z_{0}\right)$ is a collection of compact 1-dimensional submanifolds that can only have boundary points, at the boundary of the moduli space. Since it avoids the bubble curves, it can actually only have boundary points on $C_{\varepsilon}$. But because the Bishop family $\psi$ is a diffeomorphism, the 1-dimensional submanifold only touches $C_{\varepsilon}$ exactly once. This is a contradiction, because it means there is one component of the preimage that is a compact 1-dimensional manifold with only one boundary component.

\section{A Bishop family around elliptic singularities}

The aim of this section is to show that there exists a unique family of holomorphic curves growing out of each component $S$ of the elliptic singular set of a maximally foliated submanifold. I'm indebted to Frédéric Bourgeois for orienting me through the theory of Cauchy-Riemann operators.

The main idea consists in arranging a certain almost complex structure around $S$, where it is possible to do all required computations explicitly.

\subsection{A local model for a neighborhood of the singular set}

Let $(M, \alpha)$ denote always in this section a contact $(2 n-1)$-manifold, which has a symplectic filling $(W, \omega)$. Assume further that $M$ contains a maximally foliated 
submanifold $F$, with an elliptic singular set, and let $S$ be one component of this set. To simplify the calculation, we want to find some standard form for the neighborhood of the singular set $S$.

Proposition 4 There is a small neighborhood of the singular set $S \subset F$ in $M$ that is contactomorphic to a neighborhood of $\{0\} \times S$ in the contact manifold

$$
\left(\mathbb{D}^{3} \times T^{*} S, d z+\frac{1}{2}(x d y-y d x)+\lambda_{\text {can }}\right),
$$

where $(x, y, z)$ are the coordinates on the $3-b a l l$, and $\lambda_{\text {can }}=-\mathbf{p} \cdot d \mathbf{q}=-\sum_{j} p_{j} d q^{j}$ is the canonical $1-$ form on $T^{*} S$. In this neighborhood, the maximally foliated submanifold $F$ lies in the set $\left\{\left(x, y, 0 ; \sigma_{0}(s)\right)\right\}$, where $\sigma_{0}$ denotes the zero-section in $T^{*} S$.

Proof By our definition of an elliptic singular set, we find a neighborhood around $S$ inside $F$ that can be written as $\{(w ; s) \in \mathbb{C} \times S\}$, and the restriction of the contact form is equal to

$$
\left.\alpha\right|_{T F}=\frac{1}{2}(x d y-y d x)
$$

with $w=x+i y$.

Choose now a $d \alpha$-compatible complex structure $J$ on $\xi=\operatorname{ker} \alpha$. Note that the tangent space of $S_{w_{0}}:=\left\{w_{0}\right\} \times S$ for any fixed $w_{0} \in \mathbb{C}$ lies in the contact structure $\xi$, and secondly that $J \cdot T S_{w_{0}}$ is transverse to $F$, because if there was a nonzero vector $X \in T S_{w_{0}}$ such that $J X \in T F$, then

$$
0 \neq d \alpha(X, J X)=d x \wedge d y(X, J X)=0,
$$

which is a contradiction. Hence it follows that

$$
J \cdot\left(T S_{w_{0}}\right) \cap T F=\{0\} .
$$

Similarly, since the Reeb field $X_{\text {Reeb }}$ is transverse to the contact structure $\xi$, it follows in particular that $X_{\text {Reeb }}$ is transverse both to $J \cdot\left(T S_{w_{0}}\right)$, and (at least close to the singular set $S$ ) to the maximally foliated submanifold $F$, because $T F \leq \xi$ on $S$.

Choose now a metric $g$ on $M$ such that $J \cdot\left(T S_{w_{0}}\right) \perp F, X_{\text {Reeb }} \perp F$, and $\left\|X_{\text {Reeb }}\right\|=1$. This gives an identification for the normal bundle

$$
\nu F \cong\left\langle X_{\text {Reeb }}\right\rangle \oplus J \cdot\left(T S_{w_{0}}\right),
$$


which can be combined with the map

$$
\begin{aligned}
\left\langle X_{\text {Reeb }}\right\rangle \oplus J \cdot\left(T S_{w_{0}}\right) & \rightarrow \mathbb{R}^{3} \times T^{*} S \\
\left(x, y, \mathbf{q} ; z \cdot X_{\text {Reeb }}+J \dot{\mathbf{q}}\right) & \mapsto(x, y, z ; \mathbf{q}, g(\dot{\mathbf{q}}, \cdot)) .
\end{aligned}
$$

By using the exponential map as in the proof of the tubular neighborhood theorem, one gets a diffeomorphism from a neighborhood of $\{0\} \times S$ in $\mathbb{R}^{3} \times T^{*} S$ to a neighborhood of the singular set $S$ in $M$ such that $E:=\left\{\left(x, y, 0 ; \sigma_{0}(S)\right\}\right.$ is mapped into the submanifold $F$.

The pullback of the contact form evaluates in this model on $E$ to

$$
\left.\alpha\right|_{E}=d z+\frac{1}{2}(x d y-y d x)
$$

because $\partial_{z}$ is equal to the Reeb field, and the restriction of $\alpha$ to $F$ is equal to the second term. The $\mathbf{q}$ - and $\mathbf{p}$-directions lie at every point of $E$ in the contact structure. The 2-form $d \alpha$ is written on $E$ as

$$
\left.d \alpha\right|_{E}=d x \wedge d y+d \mathbf{q} \wedge d \mathbf{p}+\text { Rest },
$$

where "Rest" are terms pairing $d x$ and $d y$ with $\mathbf{p}$-coordinates.

In the final step, we use now an improved version of the Moser trick (as explained for example in Geiges [5, Theorem 2.24]) to find a vector field $X_{t}$ that isotopes the contact form given into the desired one $d z+\frac{1}{2}(x d y-y d x)+\lambda_{\text {can }}$. Let $\alpha_{t}, t \in[0,1]$, be the linear interpolation between both 1 -forms. Assume there is an isotopy $\psi_{t}$ defined around $S$ such that $\psi_{t}^{*} \alpha_{t}=\alpha_{0}$. The field $X_{t}$ generating this isotopy satisfies the equation

$$
\mathcal{L}_{X_{t}} \alpha_{t}+\dot{\alpha}_{t}=0 \text {. }
$$

By writing $X_{t}=H_{t} R_{t}+Y_{t}$, where $H_{t}$ is a smooth function, $R_{t}$ is the Reeb vector field of $\alpha_{t}$, and $Y_{t} \in \operatorname{ker} \alpha_{t}$, we obtain plugging then $R_{t}$ into the equation above

$$
d H_{t}\left(R_{t}\right)=-\dot{\alpha}_{t}\left(R_{t}\right) .
$$

The vector field $Y_{t}$ is completely determined by $H_{t}$, because $Y_{t}$ satisfies the equations

$$
\begin{aligned}
{ }^{\iota} Y_{t} \alpha_{t} & =0, \\
{ }^{{ }^{\prime}} d \alpha_{t} d \alpha_{t} & =-d H_{t}-\dot{\alpha}_{t},
\end{aligned}
$$

hence it suffices to find a suitable function $H_{t}$. Consider the 1-parameter family of Reeb fields $R_{t}$ as a single vector field on the manifold $[0,1] \times\left(\mathbb{R}^{3} \times T^{*} S\right)$. Since $R_{t}$ is transverse to the submanifold $N:=[0,1] \times\left(\mathbb{R}^{2} \times\{0\} \times T^{*} S\right)$ along $[0,1] \times E$, it is possible to define a solution $H_{t}$ to $d H_{t}\left(R_{t}\right)=-\dot{\alpha}_{t}\left(R_{t}\right)$, such that $\left.H_{t}\right|_{N} \equiv 0$. In fact, 
because $\left.\dot{\alpha}\right|_{E}=0$, it follows that $\left.d H_{t}\right|_{E}=0$, and so the vector field $X_{t}=H_{t} R_{t}+Y_{t}$ vanishes on $E$. Hence $X_{t}$ can be integrated on a small neighborhood of $E$, and $E$ is not moved under the flow, which finishes the proof of the proposition.

We can easily choose a compatible almost complex structure $J$ on the symplectization

$$
\left(W=\mathbb{R} \times\left(\mathbb{D}^{3} \times T^{*} S\right), \omega=d\left(e^{t}\left(d z+\frac{1}{2}(x d y-y d x)+\lambda_{\text {can }}\right)\right)\right),
$$

by observing that the Reeb field is given by $X_{\text {Reeb }}=e^{-t} \partial_{z}$, and that the kernel of $\alpha$ is spanned by $\partial_{x}+\frac{y}{2} \partial_{z}, \partial_{y}-\frac{x}{2} \partial_{z}$, and the vectors $X-\lambda_{\text {can }}(X) \partial_{z}$ for all $X \in T\left(T^{*} S\right)$. Choose a metric $g$ on $S$, and let $J_{0}$ be the almost complex structure on $T^{*} S$ constructed in Appendix B that is compatible with $d \lambda_{\text {can }}$.

With this, we can define a $J$ on $W$ by $J \partial_{t}=X_{\text {Reeb }}, J X_{\text {Reeb }}=-\partial_{t}, J\left(\partial_{x}+\frac{y}{2} \partial_{z}\right)=$ $\partial_{y}-\frac{x}{2} \partial_{z}, J\left(\partial_{y}-\frac{x}{2} \partial_{z}\right)=-\partial_{x}-\frac{y}{2} \partial_{z}$, and $J\left(X-\lambda_{\text {can }}(X) \partial_{z}\right)=J_{0} X-\lambda_{\text {can }}\left(J_{0} X\right) \partial_{z}$. The last equation can also be written as $J X=J_{0} X-e^{t} \lambda_{\text {can }}(X) \partial_{t}-\lambda_{\text {can }}\left(J_{0} X\right) \partial_{z}$.

As a matrix, the complex structure $J$ takes the form:

$$
J(t ; x, y, z ; \mathbf{q}, \mathbf{p})=\left(\begin{array}{ccccc}
0 & \frac{y}{2} e^{t} & -\frac{x}{2} e^{t} & -e^{t} & -e^{t} \lambda_{\text {can }} \\
0 & 0 & -1 & 0 & 0 \\
0 & 1 & 0 & 0 & 0 \\
e^{-t} & -\frac{x}{2} & -\frac{y}{2} & 0 & -\lambda_{\text {can }} \circ J_{0} \\
0 & 0 & 0 & 0 & J_{0}
\end{array}\right)
$$

Note that the last row and column represent linear maps from or to $T\left(T^{*} S\right)$. A lengthy computation (which becomes very easy on the singular set $S$ ) shows that this structure is compatible with $\omega$.

Proposition 5 The almost complex manifold $(W, J)$ can be mapped with a biholomorphism to

$$
\left(\mathbb{C}^{2} \times T^{*} S, i \oplus J_{0}\right)
$$

Proof The desired biholomorphism is

$$
\Phi(t, x, y, z ; \mathbf{q}, \mathbf{p})=(\tilde{t}, \tilde{x}, \tilde{y}, \widetilde{z} ; \widetilde{\mathbf{q}}, \widetilde{\mathbf{p}})=\left(-e^{-t}-\frac{x^{2}+y^{2}}{4}-F, x, y, z ; \mathbf{q}, \mathbf{p}\right),
$$

with the function

$$
F: T^{*} M \rightarrow \mathbb{R}, \quad(\mathbf{q}, \mathbf{p}) \mapsto \frac{\|\mathbf{p}\|^{2}}{2} .
$$


It brings $J$ into standard form with respect to the coordinate pairs $(\tilde{x}, \tilde{y}),(\widetilde{t}, \widetilde{z})$. More explicitly, by pulling back $J$ under the inverse of $\Phi$

$$
\Phi^{-1}(\tilde{t}, \tilde{x}, \tilde{y}, \tilde{z} ; \tilde{\mathbf{q}}, \tilde{\mathbf{p}})=(t, x, y, z ; \mathbf{q}, \mathbf{p})=\left(-\ln \left(-\tilde{t}-\frac{\tilde{x}^{2}+\tilde{y}^{2}}{4}-F\right), \tilde{x}, \tilde{y}, \tilde{z} ; \tilde{\mathbf{q}}, \tilde{\mathbf{p}}\right),
$$

ie by computing $D \Phi \cdot J \cdot D \Phi^{-1}$, we obtain the matrix

$$
D \Phi \cdot J \cdot D \Phi^{-1}=\left(\begin{array}{ccccc}
0 & 0 & 0 & -1 & -\lambda_{\text {can }}-d F \circ J_{0} \\
0 & 0 & -1 & 0 & 0 \\
0 & 1 & 0 & 0 & 0 \\
1 & 0 & 0 & 0 & d F-\lambda_{\text {can }} \circ J_{0} \\
0 & 0 & 0 & 0 & J_{0}
\end{array}\right)
$$

and since, according to Appendix $\mathrm{B}, d F \circ J_{0}=-\lambda_{\text {can }}$, this gives the desired normal form.

As just proved, the neighborhood of the singular set $S$ in $W$ can be regarded as $\mathbb{C}^{2} \times T^{*} S=\left\{\left(w_{1}, w_{2} ; \mathbf{q}, \mathbf{p}\right)\right\}$ with the almost complex structure $i \oplus J_{0}$. The contact manifold $M$ is given in this model by the set

$$
\left\{\left(w_{1}, w_{2} ; \mathbf{q}, \mathbf{p}\right) \in \mathbb{C}^{2} \times\left. T^{*} S\left|\operatorname{Re} w_{1}=-\frac{1}{4}\right| w_{2}\right|^{2}-\frac{1}{2}\|\mathbf{p}\|^{2}\right\} .
$$

The contact manifold is thus a hypersurface, represented by a graph, which is curved downward by the distance from the singular set (Figure 6). By using the form of a maximally foliated submanifold $F$ found in Proposition 4, one can write $F$ in a neighborhood of its singular set as the graph

$$
\begin{aligned}
\mathbb{C} \times S & \rightarrow \mathbb{C}^{2} \times T^{*} S \\
(w, \mathbf{q}) & \mapsto\left(-\frac{1}{4}|w|^{2}, w ; \mathbf{q}, 0\right) .
\end{aligned}
$$

\subsection{Explicit solutions for the Cauchy-Riemann operator}

With the local form obtained in the section above for elliptic singularities, it is very easy to write down explicitly a family of holomorphic disks with boundary on the maximally foliated submanifold $F$. We will then show that no other simple $J$-holomorphic curves, which can be capped off with a disk that lies in $F$, can enter this neighborhood. 


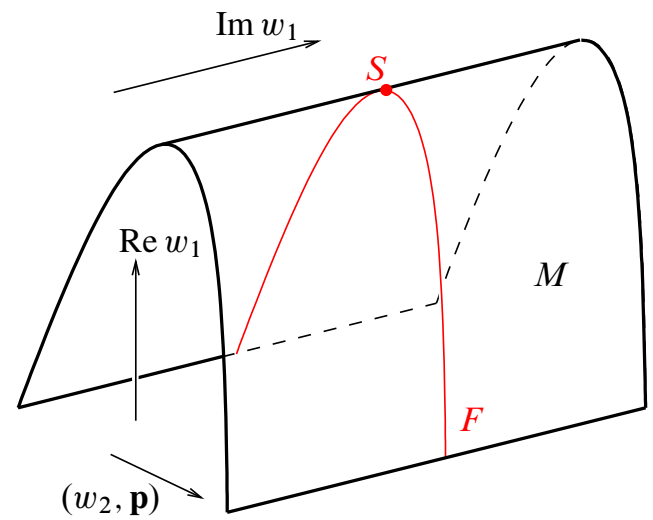

Figure 6: The contact manifold $M=\left\{\operatorname{Re} w_{1}=-\frac{1}{2}\left|w_{2}\right|^{2}-\frac{1}{4}\|\mathbf{p}\|^{2}\right\}$ appears in the standard neighborhood of $S$ like a parabola curved downwards in half of the directions.

Let in this section denote $(W, \omega)$ again a $2 n$-dimensional symplectic filling of $(M, \alpha)$, and let $U$ be the neighborhood of $S$

$$
\begin{array}{r}
U=\left\{\left(z_{1}, z_{2} ; \mathbf{q}, \mathbf{p}\right) \in \mathbb{C}^{2} \times T^{*} S \mid-C<\operatorname{Re} z_{1} \leq 0, \quad-C<\operatorname{Im} z_{1}<C,\right. \\
\left.\operatorname{Re} z_{1}+\frac{1}{4}\left|z_{2}\right|^{2}+\frac{1}{2}\|\mathbf{p}\|^{2} \leq 0\right\} \subset W
\end{array}
$$

for $C>0$ small enough ( $U$ is the half-space below the parabola in Figure 7).

Proposition 6 Let us consider the set of $J$-holomorphic curves $f: \Sigma \rightarrow W$ whose boundary sits on the maximally foliated submanifold $F$. Among those, the disks

$$
\begin{aligned}
u_{t_{0}, \mathbf{q}_{0}}: & \mathbb{D}^{2} \rightarrow \mathbb{C}^{2} \times T^{*} S \\
z & \mapsto\left(-t_{0}, 2 \sqrt{t_{0}} z ; \mathbf{q}_{0}, 0\right),
\end{aligned}
$$

for fixed $\mathbf{q}_{0} \in S$, and $t_{0} \in \mathbb{R}_{>0}$ are (up to reparametrization) the only simple curves that are completely contained in the neighborhood $U$ of the singular set $S$ just defined.

Proof That the maps $u_{t_{0}, \mathbf{q}_{0}}$ are really $J$-holomorphic disks is obvious, and that they sit on $F$ can also be checked very easily.

Assume now, there was a holomorphic curve $u: \Sigma \rightarrow W$ different from any of the solutions $u_{t_{0}, \mathbf{q}_{0}}$. If $\partial \Sigma=\varnothing$, then $u$ has to be constant, because $\omega$ is exact in the considered neighborhood. Note that the projections $\pi_{1}$ and $\pi_{2}$ of $\mathbb{C}^{2} \times T^{*} S$ onto $\mathbb{C}^{2}$ or onto $T^{*} S$ can be concatenated with the map $u$ to provide easier holomorphic curves. In particular, it follows that $u_{2}:=\pi_{2} \circ u: \Sigma \rightarrow T^{*} S$ is a $J_{0}$-holomorphic 
curve, whose boundary sits on the zero-section of the cotangent bundle. The energy of $u_{2}$ is given by

$$
E\left(u_{2}\right)=\int_{u_{2}} d \lambda_{\text {can }}=\int_{\Sigma} u_{2}^{*} d \lambda_{\text {can }}=\int_{\partial \Sigma} u_{2}^{*} \lambda_{\text {can }}=0 .
$$

Hence it follows that $u_{2}=\pi_{2} \circ u \equiv \mathbf{q}_{0}$ is a constant curve.

The other component $\pi_{1} \circ u$ of the holomorphic curve can be written as two ordinary holomorphic functions

$$
\left(f_{1}, f_{2}\right):=\pi_{1} \circ u .
$$

The first function $f_{1}: \Sigma \rightarrow \mathbb{C}$ has vanishing imaginary part on all boundary components, and hence it has vanishing imaginary part everywhere. As a consequence, it follows that the real part of $f_{1}$ is constant, as can be seen by using the Cauchy-Riemann equations, and deleting the vanishing imaginary part.

So far, we have shown that any solution is of the form $z \mapsto\left(-t_{0}, f_{2}(z) ; \mathbf{q}_{0}, 0\right)$, where $f_{2}: \Sigma \rightarrow \mathbb{C}$ is a holomorphic function, such that $\left.\left|f_{2}\right|_{\partial \Sigma}\right|^{2} \equiv 4 t_{0}$. Assume for simplicity that $t_{0}=1 / 4$, then with the maximum principle it follows that $f_{2}(\Sigma) \subset \mathbb{D}^{2}$.

For any $p \in \operatorname{int} \Sigma$, there exist holomorphic charts around $p$ and $f_{2}(p)$ such that $f_{2}$ takes the form $w \mapsto w^{k}$, but if $k \neq 1$, then $f_{2}$ is locally a branched covering, and $u$ cannot be simple. It follows that $f_{2}$ does not have any critical points, and so it is a local injective diffeomorphism in the interior of $\Sigma$. In particular, $\Sigma$ has only one boundary component, and has to be a disk $\mathbb{D}^{2}$.

The map

$$
\begin{aligned}
h_{w}: \mathbb{D}^{2} & \rightarrow \mathbb{D}^{2} \\
z & \mapsto \frac{z-w}{1-\bar{w} z}
\end{aligned}
$$

is a biholomorphism on $\mathbb{D}^{2}$, that maps $w \in \mathbb{D}^{2}$ to 0 . If we set $w=f_{2}(0)$, then the concatenation $H_{1}:=h_{w} \circ f_{2}$ is a holomorphic map from the unit disk to itself, such that $H_{1}(0)=0$. The winding number of $\left.H_{1}\right|_{\mathbb{S}^{1}}$ is still 1 .

Define now a function $H_{2}(z):=H_{1}(z) / z$, this map is holomorphic on $\mathbb{D}^{2}-\{0\}$, and can be continuously extended to 0 by setting $H_{2}(0):=H_{1}^{\prime}(0)$. This extension is also holomorphic. Furthermore, $H_{2}\left(\mathbb{S}^{1}\right) \subset \mathbb{S}^{1}$, and by the maximum principle $H_{2}\left(\mathbb{D}^{2}\right) \subset \mathbb{D}^{2}$. The winding number of $\left.H_{2}\right|_{\mathbb{S}^{1}}$ is zero, and hence it follows that $\partial_{\varphi} H_{2}\left(e^{i \varphi}\right)$ vanishes for some angle $\varphi_{0}$. With the Cauchy-Riemann equation it follows that $\partial_{r} H_{2}\left(e^{i \varphi}\right)=0$, because

$$
\partial_{\varphi} H_{2}=\left(x \partial_{y}-y \partial_{x}\right)\left(\operatorname{Re} H_{2}+i \operatorname{Im} H_{2}\right)=i \partial_{r} H_{2} .
$$


In particular it follows that the function $\left|H_{2}\right|^{2}$ has vanishing derivative at $e^{i \varphi_{0}}$, but this contradicts the boundary point lemma [6, Lemma 3.4], and hence $H_{2} \equiv$ const and $H_{1}(z)=e^{i \vartheta_{0}} z$.

The next remark allows us to apply Proposition 7 in our situation.

Remark 10 Any $J$-holomorphic disk $u$ that lies in the moduli space of curves starting at a Bishop family, can be capped off by a (topological) disk that is completely contained in the maximally foliated submanifold $F$.

Proposition 7 The only nontrivial holomorphic curves intersecting the neighborhood $U$ that can be capped off by attaching disks lying in the maximally foliated submanifold $F$ are the curves $u_{t_{0}, \mathbf{q}_{0}}$ (and their multiple covers) defined in Proposition 6.

Proof Suppose first that the restriction $\left.u\right|_{u^{-1}(U)}$ of the curve $u$ has constant ( $\left.\operatorname{Im} z_{1}\right)$ coordinate on one component of $u^{-1}(U)$. Then the whole curve $u$ is contained in $U$, because with the Cauchy-Riemann equation, it follows already that $z_{1}$ is constant on this component of $u^{-1}(U)$. If $u$ approaches the boundary of the neighborhood $U$, then either the $\mathbf{p}$ - or the $z_{2}$-coordinate have to grow, but as soon as $\operatorname{Re} z_{1}+\frac{1}{4}\left|z_{2}\right|^{2}+\frac{1}{2}\|\mathbf{p}\|^{2}$ vanishes, $u$ touches the contact manifold $M$, so that $u$ is trapped by the hypersurface $M$ inside $U$.

By Proposition 6, this means that the only nontrivial holomorphic curves $u$ intersecting the neighborhood $U$, having constant $\left(\operatorname{Im} z_{1}\right)$-coordinate on a component of $u^{-1}(U)$, are the disks $u_{t_{0}, \mathbf{q}_{0}}$ given above (and their multiple covers).

Assume now that a curve $u$ enters $U$, but does not have constant $\left(\operatorname{Im} z_{1}\right)$-coordinate. To disprove the existence of $u$, we will use an intersection argument similar to the classical one in dimension 4.

Consider for every $c=c_{x}+i c_{y} \in \mathbb{C}$ the submanifold (as drawn in Figure 7)

$$
A_{c}:=\left(\left\{x+i c_{y} \in \mathbb{C} \mid x \geq c_{x}\right\} \times \mathbb{C} \times T^{*} S\right) \cap U .
$$

Such an $A_{c}$ is the codimension 1 submanifold in $U$ obtained by taking a slice with constant imaginary $z_{1}$-coordinate and chopping off everything having smaller real $z_{1}$-coordinate than $c_{x}$. The boundary of $A_{c}$ is composed by two smooth manifolds: one of them is

$$
B_{c}:=\left(\{c\} \times \mathbb{C} \times T^{*} S\right) \cap U
$$

which is a compact $J$-holomorphic codimension 2 submanifold. Note that $U$ is foliated by the $B_{c}$ for different values of $c \in \mathbb{C}$. 
The second part of the boundary is given by the set $A_{c} \cap M$. Since the boundary is convex, holomorphic curves can only touch $A_{c} \cap M$ at their own boundary, but since the boundary of the holomorphic curves $u$ we are considering, lies in $F, u$ will never intersect $A_{c} \cap M$ if $c_{y} \neq 0$.

Thus, we have obtained a (nonsmooth) closed manifold $\widehat{B}_{c}=\partial A_{c}$, that represents the trivial homology class in $H_{2 n-2}(W)$, and which allows us to compute the intersection number with holomorphic curves.

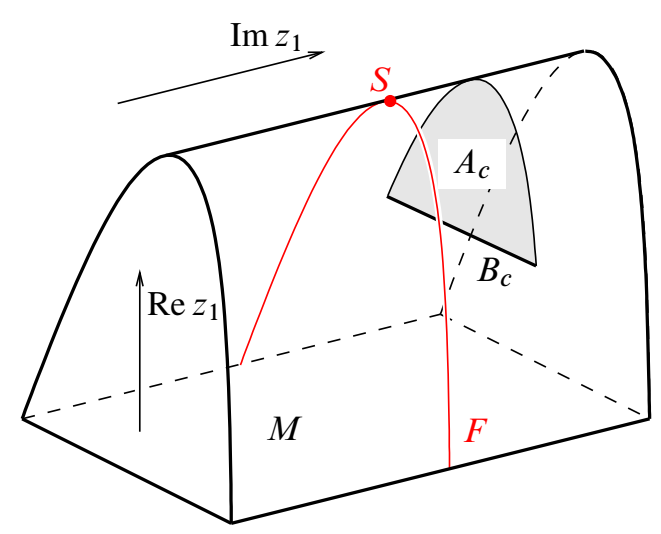

Figure 7: The neighborhood $U$ of the singular set is foliated by $J-$ holomorphic codimension 2 submanifolds $B_{c}$, which represent one part of the boundary of the slices $A_{c}$. This gives rise to an intersection argument.

Let $u$ be now any holomorphic curve that passes through the model neighborhood $U$, and whose boundary lies on the maximally foliated submanifold $F$. Assume $u$ can be capped off by attaching a disk in $F$, and denote $u$ together with its attached disk by $\widehat{u}$.

If $u$ has nonconstant imaginary $z_{2}$-coordinate in the model neighborhood $U$, then there is (by Sard's Theorem) a submanifold $B_{c}$ (with $\operatorname{Im} c \neq 0$ ) that intersects $u$ transversally at a discrete set of points. The two homology classes $[\widehat{u}]$ and $\left[\widehat{B}_{c}\right]$ have positive intersection number, because the only intersections between both classes lie in the subset, where both are represented by $J$-holomorphic submanifolds. But a positive intersection number is not possible, since $\left[\widehat{B}_{c}\right]$ represents the trivial homology class in $H_{2 n-2}(W)$.

\subsection{Expected dimension for the bishop family}

The expected dimension for the solution space of the Cauchy-Riemann operator at a holomorphic disk $u$, whose boundary lies on a totally real submanifold $F$, is given by 
the formula (see McDuff and Salamon [10, Theorem C.1.10])

$$
\operatorname{index} \bar{\partial}_{J}=\frac{\operatorname{dim} W}{2}+\mu\left(u^{*} T W, u^{*} T F\right),
$$

where we have used that the Euler characteristic of a disk is $\chi\left(\mathbb{D}^{2}\right)=1$. Here $\mu\left(E_{\mathbb{C}}, E_{\mathbb{R}}\right)$ denotes the Maslov index of a complex vector bundle $E_{\mathbb{C}}$ over a disk $\mathbb{D}^{2}$ with respect to a totally real subbundle $E_{\mathbb{R}} \leq\left. E_{\mathbb{C}}\right|_{\mathbb{S}^{1}}$ over the boundary of $\mathbb{D}^{2}$.

To obtain the expected dimension of the moduli space, we have to subtract 2 , which corresponds to the dimension of the automorphism group of the holomorphic unit disk with one marked point on the boundary.

Proposition 8 The Maslov index $\mu\left(u^{*} T W, u^{*} T F\right)$ is equal to 2 for any of the holomorphic disks

$$
\begin{aligned}
u & : \mathbb{D}^{2} \hookrightarrow \mathbb{C}^{2} \times T^{*} S \\
z & \mapsto\left(-t_{0}, 2 \sqrt{t_{0}} z ; \mathbf{q}_{0}, 0\right)
\end{aligned}
$$

given in Proposition 6 above.

Proof We can trivialize $u^{*} T W$ by choosing the obvious complex basis

$$
u^{*} T W=\left\langle\partial_{x_{1}}, \partial_{x_{2}}, \partial_{q^{1}}, \ldots, \partial_{q^{n}}\right\rangle_{\mathbb{C}} .
$$

The totally real subbundle at the boundary of $u\left(e^{i \varphi}\right)$ is spanned by the vectors

$$
\left(\left.u\right|_{\partial \mathbb{D}^{2}}\right)^{*} T F=\left\langle\partial_{x_{2}}-\sqrt{t_{0}} \cos \varphi \partial_{x_{1}}, \partial_{y_{2}}-\sqrt{t_{0}} \sin \varphi \partial_{x_{1}}, \partial_{q^{1}}, \ldots, \partial_{q^{n}}\right\rangle_{\mathbb{R}} .
$$

This subbundle can be represented at a point $\left(-t_{0}, 2 \sqrt{t_{0}} e^{i \varphi} ; \mathbf{q}_{0}\right) \in F$ by the matrix

$$
\Lambda\left(e^{i \varphi}\right)=\left(\begin{array}{ccc}
-\sqrt{t_{0}} \cos \varphi & -\sqrt{t_{0}} \sin \varphi & 0 \\
1 & i & 0 \\
0 & 0 & \mathbb{1}
\end{array}\right)
$$

with respect to the complex basis of $u^{*} T W$. It follows that the Maslov index $\mu\left(u^{*} T W, u^{*} T F\right)$ is given by

$$
\begin{aligned}
\mu\left(u^{*} T W, u^{*} T F\right) & =\operatorname{deg} \frac{\operatorname{det} \Lambda^{2}}{\operatorname{det} \Lambda^{*} \Lambda}=\operatorname{deg} \frac{-t_{0}\left(\cos ^{2} \varphi-\sin ^{2} \varphi+2 i \sin \varphi \cos \varphi\right)}{t_{0}} \\
& =\operatorname{deg}\left(-e^{2 i \varphi}\right)=2 .
\end{aligned}
$$

The expected dimension of the moduli space $\mathcal{M}=\mathcal{M}\left(\mathbb{C}^{2} \times T^{*} S, z_{0}\right)$ is thus

$$
\operatorname{dim} \mathcal{M}=\frac{\operatorname{dim} W}{2}+2-2=\frac{\operatorname{dim} W}{2}
$$

Algebraic 83 Geometric Topology, Volume 6 (2006) 
which means that the family of solutions, we have found above are locally all solutions, if the Cauchy-Riemann operator for the given $J$ is regular. That this is indeed the case will be shown below.

\subsection{Surjectivity of the linearized Cauchy-Riemann operator}

In this section, we will show that the linearized Cauchy-Riemann operator $\bar{\partial}_{J}$ at any of the curves $u_{t_{0}, \mathbf{q}_{0}}$ from Proposition 6 is surjective. Since we have shown that these disks are the only holomorphic curves contained in our model neighborhood, we can apply [10, Remark 3.2.3]. Thus it will be enough to perturb $J$ outside the model neighborhood to obtain a regular Cauchy-Riemann operator for the whole moduli space.

Proposition 9 The linearized Cauchy-Riemann operator $D_{u} \bar{\partial}_{J}$ is surjective at any of the disks $u=u_{t_{0}, \mathbf{q}_{0}}$ specified in Proposition 6.

Proof Instead of checking that $D_{u} \bar{\partial}_{J}$ is surjective, we will compute the dimension of its kernel, and see that it coincides with the index of the operator. It then follows that the cokernel must be trivial.

Write again $W$ for the neighborhood $\mathbb{C}^{2} \times T^{*} S$, and let $v+i w$ be the standard coordinates on the disk. The linearized Cauchy-Riemann operator at a disk $u: \mathbb{D}^{2} \rightarrow W$ is given by

$$
\left(D_{u} \bar{\partial}_{J}\right) \dot{u}=\frac{\partial \dot{u}}{\partial v}+J(u) \frac{\partial \dot{u}}{\partial w}+\left(\left.\frac{d}{d s}\right|_{s=0} J(u+s \dot{u})\right) \frac{\partial u}{\partial w},
$$

where $\dot{u}$ denotes a section of $u^{*} T W$, which restricts to a section of $u^{*} T F$ along the boundary of $\mathbb{D}^{2}$. To compute the kernel, we have to find all solutions $\dot{u}$ of the equation $\left(D_{u} \bar{\partial}_{J}\right) \dot{u}=0$.

Note that the linearized Cauchy-Riemann equation simplifies to

$$
\frac{\partial \dot{u}}{\partial v}+J(u) \frac{\partial \dot{u}}{\partial w}=0,
$$

because the derivative of the almost complex structure can be dropped: for the $\mathbb{C}^{2}$ part, this is obvious, because the complex structure is constant; for the $T^{*} S$ part, this follows, because we multiply with $\partial_{w} u$, which vanishes in the cotangent bundle for any of the curves $u=u_{t_{0}, \mathbf{q}_{0}}$ from Proposition 6 . 
Take a canonical chart $\left\{\left(z_{1}, z_{2} ; \mathbf{q}, \mathbf{p}\right)\right\}$ in $W$ containing the disk $u$, and use this to express $\dot{u}$ as

$$
\begin{aligned}
\dot{u}: \mathbb{D}^{2} \rightarrow \mathbb{C}^{2} \times \mathbb{R}^{2 n-4} & \\
v+i w & \mapsto\left(\dot{z}_{1}, \dot{z}_{2} ; \dot{\mathbf{q}}, \dot{\mathbf{p}}\right) .
\end{aligned}
$$

The boundary conditions give $\left.\operatorname{Im}\left(\dot{z}_{1}\right)\right|_{\mathbb{S} 1} \equiv 0,\left.\operatorname{Re}\left(\dot{z}_{1}\right)\right|_{\mathbb{S} 1}=\frac{1}{4}\left(z_{2} \overline{\dot{z}}_{2}+\bar{z}_{2} \dot{z}_{2}\right)$ and $\left.\dot{\mathbf{p}}\right|_{\mathbb{S} 1} \equiv 0$.

The linearized Cauchy-Riemann equation decomposes into two independent equations, one on $\mathbb{C}^{2}$, and one on $T^{*} S$. We will first analyze the part on $T^{*} S$. The holomorphic disk at which we are linearizing is just the constant map $\left(\mathbf{q}_{0}, 0\right) \in T^{*} S$. We can assume the chart $\left(q^{1}, \ldots, q^{n-2}, p_{1}, \ldots, p_{n-2}\right)$ to be induced by a geodesic normal chart $\left(q^{1}, \ldots, q^{n-2}\right)$ around $\mathbf{q}_{0} \in S$. The explicit form of $J_{0}$ in such a chart can be found in the proof of Theorem 13 in Appendix B. The linearized Cauchy-Riemann equation becomes

$$
\frac{\partial \dot{\mathbf{q}}}{\partial v}-\frac{\partial \dot{\mathbf{p}}}{\partial w}=0 \quad \text { and } \quad \frac{\partial \dot{\mathbf{p}}}{\partial v}+\frac{\partial \dot{\mathbf{q}}}{\partial w}=0 .
$$

This gives rise to the equation $\Delta \dot{\mathbf{p}}=0$, which in turn together with the boundary condition implies that $\dot{\mathbf{p}} \equiv 0$. By plugging this into the Cauchy-Riemann equation, it finally follows that $\dot{\mathbf{q}} \equiv$ const. That means we find $n-2$ degrees of freedom from the $T^{*} S$ part.

The equation for $z_{1}$ is the standard Cauchy-Riemann equation, ie

$$
\frac{\partial \dot{z}_{1}}{\partial v}+i \frac{\partial \dot{z}_{1}}{\partial w}=0
$$

or split into real part and imaginary part (with $\dot{z}_{1}=\dot{x}_{1}+i \dot{y}_{1}$ )

$$
\frac{\partial \dot{x}_{1}}{\partial v}-\frac{\partial \dot{y}_{1}}{\partial w}=0 \quad \text { and } \quad \frac{\partial \dot{y}_{1}}{\partial v}+\frac{\partial \dot{x}_{1}}{\partial w}=0 .
$$

Combining these equations, we obtain $\Delta \dot{y}_{1} \equiv 0$, and together with the boundary condition, it follows that $\dot{y}_{1} \equiv 0$. Using this result again in the linearized CauchyRiemann equation, we obtain $\dot{x}_{1} \equiv$ const.

The function $\dot{z}_{2}$ is a holomorphic function on the unit disk, ie we can write $\dot{z}_{2}$ down as a power series

$$
\dot{z}_{2}(v+i w):=\sum_{k=0}^{\infty} a_{k}(v+i w)^{k}
$$


or when restricted to the boundary $\mathbb{S}^{1}=\left\{e^{i \varphi}\right\}$ and assuming that $a_{k}=b_{k}+i c_{k}$,

$$
\dot{z}_{2}\left(e^{i \varphi}\right):=\sum_{k=0}^{\infty}\left(b_{k}+i c_{k}\right) e^{i k \varphi} .
$$

The function $\dot{z}_{1} \equiv$ const was coupled to $\dot{z}_{2}$ by the boundary condition

$$
\left.\dot{z}_{1}\right|_{\mathbb{S} 1}=\left.\frac{1}{4}\left(z_{2} \overline{\dot{z}}_{2}+\bar{z}_{2} \dot{z}_{2}\right)\right|_{\mathbb{S} 1},
$$

and by using that $z_{2}=\sqrt{t_{0}}(v+i w)$ (after a reparametrization), it follows that

$$
\begin{aligned}
\mathrm{const} & \equiv e^{-i \varphi} \sum_{k=0}^{\infty}\left(b_{k}+i c_{k}\right) e^{i k \varphi}+e^{i \varphi} \sum_{k=0}^{\infty}\left(b_{k}-i c_{k}\right) e^{-i k \varphi} \\
& =\sum_{k=0}^{\infty}\left(b_{k}+i c_{k}\right) e^{i(k-1) \varphi}+\sum_{k=0}^{\infty}\left(b_{k}-i c_{k}\right) e^{-i(k-1) \varphi} \\
& =\sum_{k=-1}^{\infty}\left(b_{k+1}+i c_{k+1}\right) e^{i k \varphi}+\sum_{k=-1}^{\infty}\left(b_{k+1}-i c_{k+1}\right) e^{-i k \varphi} \\
= & 2 \sum_{k=-1}^{\infty} b_{k+1} \cos k \varphi-2 \sum_{k=-1}^{\infty} c_{k+1} \sin k \varphi \\
= & 2 b_{1}-2 c_{1}+2\left(b_{0}+b_{2}\right) \cos \varphi+2\left(c_{0}-c_{2}\right) \sin \varphi \\
& +2 \sum_{k=2}^{\infty}\left(b_{k+1} \cos k \varphi-c_{k+1} \sin k \varphi\right) .
\end{aligned}
$$

And hence the coefficients $a_{0}$ and $a_{1}$ can be chosen arbitrarily, $a_{2}=-\bar{a}_{0}$, and $a_{3}=a_{4}=\ldots=0$ must all vanish. The function $\dot{z}_{2}$ is thus given by $\dot{z}_{2}=a_{0}+a_{1} z-\bar{a}_{0} z^{2}$, and so the $\mathbb{C}^{2}$ part contributes a 4 -dimensional kernel to the linearized CauchyRiemann operator.

Since both parts are independent, the dimension of the kernel of the linearized CauchyRiemann operator is equal to $4+\operatorname{dim} S=\operatorname{dim} W / 2+2$, which is equal to the Fredholm index, as we wanted to show.

\section{Bubbling off analysis}

The moduli spaces of holomorphic disks, whose boundary lies on a compact totally real submanifold, and which have a uniform energy bound, are compact, provided one includes two different types of bubbling: the curves can either form a bubble at 
their boundary or a bubble in their interior. Though a maximally foliated submanifold $F$ with elliptic singularities is not compact, if one removes the singularities (or not totally real, if one does not remove the singularities), we still have compactness for the moduli spaces coming from Bishop families, because we have proved in Proposition 7 that elliptic singularities have a small neighborhood, which blocks out every curve with exception of a Bishop family. This confines the movement of all other disks to a compact totally real submanifold. But first, we will show that the energy for every curve of the moduli space is bounded.

Proposition 10 Let $\left(M^{2 n-1}, \alpha\right)$ be a closed contact manifold that has a symplectic filling $(W, \omega)$. Assume that $M$ contains a plastikstufe $\mathcal{P S}(S)$. Let $u:\left(\mathbb{D}^{2}, \mathbb{S}^{1}\right) \rightarrow$ $(W, \mathcal{P S}(S))$ be a holomorphic disk that lies in the same moduli space as the Bishop family found in Proposition 6.

There is a constant $C$, which only depends on $\left.\alpha\right|_{T \mathcal{P S}(S)}$ that bounds the energy

$$
E(u)=\int_{u} \omega
$$

of any such disk.

Proof The disk $u$ is in $(W, \mathcal{P} S(S))$ homotopic to a point, hence the energy $E(u)$ of $u$ can be obtained by

$$
E(u)=\int_{u} \omega=\int_{\partial u} \alpha .
$$

Let now $P$ denote the submanifold $\mathcal{P S}(S)-(S \cup \partial \mathcal{P S}(S))$, ie the plastikstufe with its boundary and the interior singularity removed. Note that the standard leaves of the foliation of $\mathcal{P} \mathcal{S}(S)$ can be labeled bijectively with elements $e^{i \varphi} \in \mathbb{S}^{1}$, and in fact there is a smooth surjective map

$$
\vartheta: P \rightarrow \mathbb{S}^{1}
$$

such that $\vartheta(p)=\vartheta(q)$ if and only if, $p$ and $q$ lie on the same leaf of the foliation. The differential $d \vartheta$ can be regarded as a 1 -form on $P$. Note that there is a unique smooth function $f: P \rightarrow \mathbb{R}_{>0}$ such that

$$
\alpha=f d \vartheta
$$

because the kernel of both 1 -forms agree on $P$.

The boundary of every holomorphic disk $u$ in our moduli space is transverse to the foliation, because if the tangent direction of $u$ at a boundary point $z \in \partial \mathbb{D}^{2}$ lay in ker $\alpha$, then by our definition of $J$ the whole tangent space $T_{u(z)} u$ would be tangent to 
$M$. But this contradicts Corollary 3 , and hence it follows that $\left.\vartheta \circ u\right|_{\mathbb{S}^{1}}$ makes exactly one turn, or expressed in a different way,

$$
\int_{\partial u} d \vartheta=2 \pi
$$

For every point $p_{0}$ on $\partial \mathcal{P S}(S)$, we find a chart $U \subset \mathbb{R}_{\leq 0} \times \mathbb{R}^{N-1}(N:=\operatorname{dim} \mathcal{P} \mathcal{S}(S))$ mapping $p_{0}$ to 0 such that the intersection of the leaves with $U$ is given by the planes

$$
F_{x}=\left(\{x\} \times \mathbb{R}^{N-1}\right) \cap U
$$

for $x \leq 0$, and $F_{0}$ corresponds to the boundary $\partial \mathcal{P} \mathcal{S}(S) \cap U$. The local picture alone does not allow us to see, which two sheets are contained in the same leaf (see Figure 8), but since the leaves in $P$ approach the boundary of the plastikstufe asymptotically, it follows that for every leaf, there is a monotonous sequence $a_{k}$ converging to 0 such that all $F_{a_{k}}$ lie in the same global leaf (and such that every other hyperplane $F_{b}$ lies in another leaf).

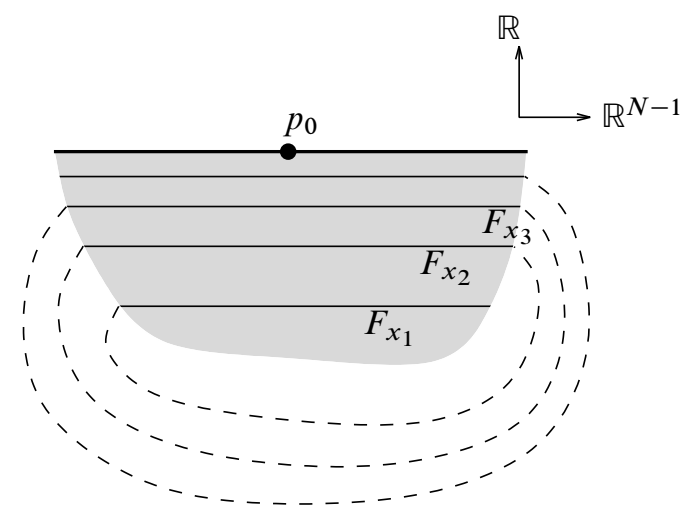

Figure 8: In a neighborhood of a point $p_{0} \in \mathcal{P} \mathcal{S}(S)$, the leaves can be represented by horizontal hyperplanes $F_{x}$. The global shape of the foliation in $P$ connects certain $F_{x}$.

Since $\partial \mathcal{P} \mathcal{S}(S)$ is compact, we can cover the whole boundary of the plastikstufe by using only a finite number of such charts $\left\{U_{1}, \ldots, U_{n_{0}}\right\}, n_{0} \in \mathbb{N}$. On each chart $U_{k}$, $\alpha$ can be written as $g_{k} d x_{1}$, where $g_{k}: U_{k} \rightarrow \mathbb{R}$ is a smooth function. In particular, $\left|g_{k}\right|$ is bounded by a number $c_{k}$.

Algebraic $8 \mathcal{G}$ Geometric Topology, Volume 6 (2006) 
For any smooth path $\gamma$ in $U_{k}$ connecting $F_{a}$ with $F_{b}$ crossing each leaf transversely in increasing direction, we can estimate the integral by

$$
\int_{\gamma} \alpha \leq c_{k}|b-a|
$$

The intersection $\partial u \cap U_{k}$, where $\partial u$ is the boundary of a $J$-holomorphic disk, gives a collection of paths $\gamma_{j} \subset U_{k}$. But since $\partial u$ crosses every global leaf in the plastikstufe once, we can order the segments of paths in such a way that the end point of $\gamma_{j}$ lies on $F_{b_{j}}$, and the segment $\gamma_{j+1}$ starts on $F_{a_{j+1}}$ with $b_{j}<a_{j+1}$. Hence the total estimate gives

$$
\int_{\partial u \cap U_{k}}=\sum_{j} \int_{\gamma_{j}} \alpha \leq c_{k} \sum_{j}\left|b_{j}-a_{j}\right|<c_{k} L_{k},
$$

where $L_{k}$ is the length of $U_{k}$ in $x_{1}$-direction.

Finally cover now the whole plastikstufe with sets $\left\{K, U_{1}, \ldots, U_{n_{0}}\right\}$, where $K$ is a compact set that never touches the boundary of the plastikstufe.

The energy of a holomorphic disk $u$ in our moduli space with boundary $\partial u$ can now be bounded by a number $C$ in the following way:

$$
E(u)=\int_{\partial u} \alpha \leq \int_{\partial u \cap K} \alpha+\sum_{k=1}^{n_{0}} \int_{\partial u \cap U_{k}} \alpha \leq \int_{\partial u \cap K} \alpha+\sum_{k=1}^{n_{0}} c_{k} L_{k} .
$$

The energy of the segments of $\partial u$ contained in $K$ can be estimated by

$$
\int_{\partial u \cap K} \alpha=\int_{\partial u \cap K} f d \vartheta \leq \sup _{p \in K} f(p) \int_{\partial u \cap K} d \vartheta \leq 2 \pi \sup _{p \in K} f(p) .
$$

By setting $C:=2 \pi \sup _{K} f+\sum_{k=1}^{n_{0}} c_{k} L_{k}$, we obtain a uniform estimate for all disks in our moduli space.

Proposition 11 Let $\left(M^{2 n-1}, \alpha\right)$ be a closed contact manifold that has a symplectic filling $(W, \omega)$, and assume that $M$ contains a plastikstufe $\mathcal{P} \mathcal{S}(S)$.

Let $u_{k}: \mathbb{D}^{2} \rightarrow W$ be a sequence of holomorphic disks, whose boundary lies in the plastikstufe with fixed linking number $\operatorname{lk}\left(\partial u_{k}, S\right)=1$. The sequence $u_{k}$ has a subsequence that converges either to a constant map, whose image lies in the singular set $S$, or to a simple smooth holomorphic disk and a finite number of bubble spheres.

Proof Assume first, there was a subsequence of disks $\left(u_{k_{l}}\right)_{l}$ coming arbitrarily close to the singular set. By Proposition 7, it follows that for $k_{l}$ sufficiently large, the disks 
$u_{k_{l}}$ lie in the Bishop family, and then $\left(u_{k_{l}}\right)_{l}$ has a further subsequence that converges to a point of $S$.

If all the disks stay at a finite distance $C>0$ from $S$, then the boundary of all disks is contained in a compact subset of a totally real submanifold, and hence by Gromov compactness, there is a subsequence $\left(u_{k_{l}}\right)_{l}$ that converges to a bubbled curve.

One possible type of bubbles that could occur, are disks growing at the boundary of the family $\left(u_{k_{l}}\right)_{l}$. Let

$$
\vartheta: \mathcal{P S}(S)-(S \cup \partial \mathcal{P} \mathcal{S}(S)) \rightarrow \mathbb{S}^{1}
$$

be the function already defined in the proof of Proposition 10. Since the boundary of every disk $u_{k}$ is transverse to the foliation, and the linking number is 1 , we can define smooth bijective maps $f_{k}:=\left.\vartheta \circ u_{k}\right|_{\mathbb{S}^{1}}: \partial \mathbb{D}^{2} \rightarrow \mathbb{S}^{1}$. The sequence $\left(u_{k_{l}}\right)_{l}$ converges to a continuous map $u_{\infty}: \mathbb{D}^{2} \rightarrow W$ in $C^{0}$-norm, and $\left(f_{k_{l}}\right)_{l}$ converges then to $f_{\infty}:=\left.\vartheta \circ u_{\infty}\right|_{\mathbb{S} 1}$. The map $f_{\infty}$ is continuous, monotonous, and has degree 1 . It follows that the only way to split $u_{\infty}$ into subbubbles is by assuming that one of the bubbles is constant at its boundary, which also implies by the same energy argument as above that the bubble is constant.

This means that the only type of bubbling, which is allowed, is bubbling in the interior of the disk. In this situation, all the bubbles are spherical. The base of the bubble tree is a simple disk $u_{0}$, because the restriction to the boundary of $u_{0}$ is by the argument above injective.

Definition Let $A$ be a subset of a manifold $M$. We say that $A$ has at most dimension $n$, if there is an $n$-dimensional manifold $X$ (with finite amount of components) and a smooth map $f: X \rightarrow M$ with closed image such that $A \subset f(X)$.

Remark 11 To apply the general theory of moduli spaces of $J$-holomorphic curves, we always assume regularity of the Cauchy-Riemann operator for every curve in $\mathcal{M}\left(W, \mathcal{P} \mathcal{S}(S), z_{0}\right)$ and for all the bubble trees considered in the proof of the next proposition. Below we will briefly sketch the argument, why this is indeed possible.

The almost complex structure $J$ on $W$ is first defined only in a neighborhood of the singular set $S$ of $\mathcal{P} \mathcal{S}(S)$ as explained in Section 3.4. In this neighborhood, regularity works as proved for $\mathcal{M}\left(W, \mathcal{P S}(S), z_{0}\right)$ and since no holomorphic spheres can enter the domain, no problem occurs.

In a second step $J$ is extended to a small collar neighborhood of $M$, such that $J$ is compatible with the convex boundary as defined in Section 0. Again holomorphic 
spheres pose no problem, and since all holomorphic disks in our moduli space are simple, by perturbing $J$, one can achieve regularity for all disks contained in the collar.

In the last step, $J$ is finally extended over the rest of the symplectic manifold $W$, now only requiring that $J$ is compatible with $\omega$. For disks, one could suspect a difficulty, because the boundary part of the disk lies in the collar where $J$ has already been defined, but [10, Remark 3.2.3] tells us that, regularity for these curves can be achieved even by perturbing $J$ only in the interior of $W$. For the bubble trees, we also obtain by perturbations of $J$ regularity as explained in [10].

Proposition 12 To compactify the moduli space $\mathcal{M}\left(W, \mathcal{P S}(S), z_{0}\right)$, one has to add bubbled curves. The image of these bubbled curves under the evaluation map $\mathrm{ev}_{z_{0}}$ has at most dimension $n-2$, where $\operatorname{dim} W=2 n$.

Proof The standard way to treat bubbled curves consists in considering them as elements in a bubble tree: here such a tree is composed by a simple holomorphic disk $u_{0}:\left(\mathbb{D}^{2}, \mathbb{S}^{1}\right) \rightarrow(W, \mathcal{P} \mathcal{S}(S))$ and holomorphic spheres $u_{1}, \ldots, u_{k^{\prime}}: \mathbb{S}^{2} \rightarrow W$. These holomorphic curves are connected to each other in a certain way. We formalize this relation by saying that the holomorphic curves are vertices in a tree, ie in a connected graph without cycles. We denote the edges of this graph by $\left\{u_{i}, u_{j}\right\}, 0 \leq i<j \leq k^{\prime}$.

Now we assign to any edge two nodal points $z_{i j}$ and $z_{j i}$, the first one in the domain of the bubble $u_{i}$, the other one in the domain of $u_{j}$, and we require that $\operatorname{ev}_{z_{i j}}\left(u_{i}\right)=$ $\mathrm{ev}_{z_{j i}}\left(u_{j}\right)$. For technical reasons, we also require nodal points on each holomorphic curve to be pairwise distinct. To include into the theory, trees with more than one bubble connected at the same point to a holomorphic curve, we add "ghost bubbles". These are constant holomorphic spheres inserted at the point where several bubbles are joined to a single curve. Now all the links at that point are opened and reattached at the ghost bubble. Ghost bubbles are the only constant holomorphic spheres we allow in a bubble tree.

The aim is to give a manifold structure to these bubble trees. Unfortunately this is in general not always possible, because already for a single sphere, one can only obtain regularity of the Cauchy-Riemann operator, if the sphere is simple.

Instead, we note that the image of every bubble tree is equal to the image of a simple bubble tree, that means, to a tree, where every holomorphic sphere is simple and any two spheres have different image. Since we are only interested in the image of the evaluation map on the bubble trees, it is for our purposes equivalent to consider the simple bubble tree instead of the original one. The disk $u_{0}$ is always simple, and does not need to be replaced by another simple curve. 
Let $u_{0}, u_{1}, \ldots, u_{k^{\prime}}$ be the holomorphic curves composing the original bubble tree, and let $A_{i} \in H_{2}(W)$ be the homology class represented by the holomorphic sphere $u_{i}$. The simple tree is composed by $u_{0}, v_{1}, \ldots, v_{k}$ such that for every $u_{j}$ there is a bubble sphere $v_{i_{j}}$ with

$$
u_{j}\left(\mathbb{S}^{2}\right)=v_{i_{j}}\left(\mathbb{S}^{2}\right)
$$

and in particular $A_{j}=m_{j} B_{i_{j}}$, where $B_{i_{j}}=\left[v_{i_{j}}\right] \in H_{2}(W)$ and $m_{j} \geq 1$ is an integer. Denote the sum $\sum_{j=1}^{k^{\prime}} A_{j}$ by $A$ and the sum $\sum_{i=1}^{k} B_{i}$ by $B$. Below we will now compute the dimension of this simple bubble tree.

The initial bubble tree $u_{0}, u_{1}, \ldots, u_{k^{\prime}}$ is the limit of a sequence in the moduli space $\mathcal{M}\left(W, \mathcal{P S}(S), z_{0}\right)$. Hence the connected sum $u_{\infty}:=u_{0} \sharp \ldots \sharp u_{k^{\prime}}$ is, as element of $\pi_{2}(W, \mathcal{P S}(S))$, homotopic to a disk $u$ in the bishop family, and the Maslov indices

$$
\mu(u):=\mu\left(u^{*} T W, u^{*} T \mathcal{P} \mathcal{S}(S)\right) \quad \text { and } \quad \mu\left(u_{\infty}\right):=\mu\left(u_{\infty}^{*} T W, u_{\infty}^{*} T \mathcal{P S}(S)\right)
$$

have to be equal. By Proposition 8 and standard rules for the Maslov index, we obtain

$$
2=\mu(u)=\mu\left(u_{\infty}\right)=\mu\left(u_{0}\right)+\sum_{j=1}^{k^{\prime}} 2 c_{1}\left(\left[u_{j}\right]\right)=\mu\left(u_{0}\right)+2 c_{1}(A) .
$$

The dimension of the unconnected set of holomorphic curves

$$
\mathcal{M}_{\left[u_{0}\right]}\left(W, \mathcal{P} \mathcal{S}(S), z_{0}\right) \times \prod_{j=1}^{k} \mathcal{M}_{B_{j}}(W)
$$

for the simple bubble tree is

$$
\begin{aligned}
\left(n+\mu\left(u_{0}\right)\right)+\sum_{j=1}^{k} 2\left(n+c_{1}\left(B_{j}\right)\right) & =n+2-2 c_{1}(A)+2 n k+\sum_{j=1}^{k} 2 c_{1}\left(B_{j}\right) \\
& =n+2+2 n k+2\left(c_{1}(B)-c_{1}(A)\right)
\end{aligned}
$$

In the next step, we want to consider the subset of connected bubbles, ie we choose a total of $k$ pairs of nodal points, which then have to be pairwise equal under the evaluation map. The nodal points span a manifold

$$
Z(2 k) \subset\left\{(1, \ldots, 2 k) \rightarrow \mathbb{D}^{2} \dot{\cup} \mathbb{S}^{2} \dot{\cup} \ldots \dot{\cup} \mathbb{S}^{2}\right\}
$$


of dimension $4 k$. The dimension reduction comes from requiring that the evaluation map

$$
\text { ev: } \mathcal{M}_{\left[u_{0}\right]}\left(W, \mathcal{P S}(S), z_{0}\right) \times \prod_{j=1}^{k} \mathcal{M}_{B_{j}}(W) \times Z(2 k) \rightarrow W^{2 k}
$$

sends pairs of nodal points to the same image in the symplectic manifold. By regularity and transversality of the evaluation map to the diagonal submanifold $\Delta(k) \hookrightarrow W^{2 k}$, the dimension of the space of holomorphic curves is reduced by the codimension of $\triangle(k)$, which is $2 n k$.

As a last step, we have to take the quotient by the automorphism group to obtain the moduli space. The dimension of the automorphism group is $6 k+2$ (the last term corresponds to the automorphism group of the holomorphic disk with one marked point on its boundary). Hence the dimension of the total moduli space is

$$
\begin{aligned}
n+2+2 n k+2\left(c_{1}(B)-c_{1}(A)\right)+4 k-2 n k-(6 k+2) \\
=n-2 k+2\left(c_{1}(B)-c_{1}(A)\right) \geq n-2 k .
\end{aligned}
$$

The inequality holds because by the assumption of semipositivity, all the Chern classes are nonnegative on holomorphic spheres, and all coefficients $n_{j}$ in the difference $c_{1}(B)-c_{1}(A)=\sum_{j} c_{1}\left(B_{j}\right)-\sum_{i} c_{1}\left(A_{i}\right)=\sum_{j} c_{1}\left(B_{j}\right)-\sum_{i} m_{i} c_{1}\left(B_{j_{i}}\right)=$ $\sum_{j} n_{j} c_{1}\left(B_{j}\right)$ are nonpositive integers.

\section{Applications}

\subsection{Exotic contact structures on $\mathbb{R}^{2 n-1}$}

A contact structure on $\mathbb{R}^{2 n-1}$ containing a plastikstufe is obviously exotic, because it cannot embed into the standard sphere $\left(\mathbb{S}^{2 n-1}, \alpha_{0}\right)$. This application is due to Chekanov and Gromov $\left[8,2.4 . D_{2}^{\prime}\right.$.(c) $]$.

Instead of using the most general setup, we will just give one example. Bates and Peschke [1] have constructed an exotic symplectic structure $\omega=-d \lambda$ (see also $\left[9\right.$, Example 13.8]) on $\mathbb{R}^{4}$ that contains a Lagrangian torus $\mathbb{T}^{2}$ such that $\left.\lambda\right|_{\mathbb{T}^{2}} \equiv 0$. Let $\alpha_{-}$be an overtwisted contact structure on $\mathbb{R}^{3}$. Then

$$
\left(\mathbb{R}^{7}=\mathbb{R}^{3} \times \mathbb{R}^{4}, \alpha_{-}+\lambda\right)
$$

is an exotic contact structure, because it contains the plastikstufe $\mathcal{P S}\left(\mathbb{T}^{2}\right)$. To the author's knowledge, there is until now, no other way to distinguishing these contact 
structures from the standard one: classical invariants fail to do so, and contact homology on open manifolds has not yet been rigorously developed.

Other examples of exotic contact structures on $\mathbb{R}^{2 n-1}$ were known before (eg Muller shows that the symplectization of an exotic $\mathbb{R}^{5}$ she constructs, contains a Lagrangian sphere [11]), but with the plastikstufe it is easy to construct examples in any dimension (embed the neighborhood of a plastikstufe into $\mathbb{R}^{2 n-1}$, and use the $h$-principle to extend the contact structure over the whole Euclidean space).

\subsection{Filling with holomorphic curves in higher dimensions}

In this section, we will apply the ideas in the proof of Theorem 1 to an example not directly related to the plastikstufe. This example can be seen as a generalization of $\left[8,2.4 . \mathrm{D}_{1}^{\prime}\right]$ to higher dimensions, and the main difficulty consists again in finding a replacement for positivity of intersections.

Consider a closed contact $(2 n-1)$-manifold $(M, \alpha)$, which is the convex boundary of a semipositive symplectic manifold $(W, \omega)$. Any Darboux chart $U \subset M$ contains subsets contactomorphic to $\mathbb{S}^{2 n-1}-\{(0, \ldots, 0,1)\}$ with the standard contact form

$$
\alpha_{0}=\sum_{j=1}^{n}\left(x_{j} d y_{j}-y_{j} d x_{j}\right),
$$

where $z_{j}=x_{j}+i y_{j}$ are complex coordinates of $\mathbb{C}^{n}$, and $\mathbb{S}^{2 n-1}$ is embedded in the standard way into $\mathbb{C}^{n}$. Note that the canonical $\mathrm{SO}(n)$-action on $\mathbb{C}^{n}$ (ie the one by matrix multiplication) restricts to the sphere and leaves $\alpha_{0}$ invariant.

Let $P_{t_{0}}$ be the 3-plane

$$
P_{t_{0}}=\left\{\left(x+i y, z+i t_{0}, 0, \ldots, 0\right) \mid x, y, z \in \mathbb{R}\right\} .
$$

Denote the intersection $P_{t_{0}} \cap \mathbb{S}^{2 n-1}$ by $\mathbb{S}_{t_{0}}^{2}$. It is a 2 -sphere (if $t_{0} \in(-1,1)$ ), and the foliation induced by $\alpha_{0}$ has only two singularities, at the north pole $N=$ $\left(0, \sqrt{1-t_{0}^{2}}+i t_{0}, 0, \ldots, 0\right)$ and the south pole $S=\left(0,-\sqrt{1-t_{0}^{2}}+i t_{0}, 0, \ldots, 0\right)$. Every leaf circles down from $N$ to $S$.

Now we consider the flow-out $F_{t_{0}}:=\mathrm{SO}(n-1) \cdot \mathbb{S}_{t_{0}}^{2}$ obtained by taking the set of all $\mathrm{SO}(n-1)$-orbits, where we use the embedding

$$
\begin{aligned}
\mathrm{SO}(n-1) & \hookrightarrow \mathrm{SO}(n) \\
A & \mapsto\left(\begin{array}{ll}
1 & 0 \\
0 & A
\end{array}\right) .
\end{aligned}
$$

Algebraic $6 \mathcal{G}$ Geometric $\mathcal{T}$ opology, Volume 6 (2006) 
Each orbit is diffeomorphic to an $(n-2)$-sphere $\mathbb{S}^{n-2} \cong \mathrm{SO}(n-1) / \mathrm{SO}(n-2)$, and lies inside the contact structure ker $\alpha_{0}$. Hence it follows that $F_{t_{0}} \cong \mathbb{S}^{2} \times \mathbb{S}^{n-2}$ is a maximally foliated submanifold, with the two elliptic singularities $\{S\} \times \mathbb{S}^{n-2}$ and $\{N\} \times \mathbb{S}^{n-2}$. The regular leaves connect the upper singularity with the lower one. We just have shown that we can find such a maximally foliated submanifold $F_{t_{0}}$ in any Darboux chart.

By choosing now the almost complex structure from Section 3 around both elliptic singularities, and extending this to a generic $\omega$-compatible structure on $W$, we obtain around each of the poles of the sphere a Bishop family, and the aim will be to show that in fact the upper and lower family lie in the same moduli space.

In the same way, as in the proof of Theorem 1, it can be excluded that the moduli space has any boundary components apart from the Bishop ends, so the moduli space is either connected with two boundary components, one for each of the Bishop families, or it consists of two disconnected spaces, each with one boundary component (see Figure 9). If the moduli space was disconnected, then the Bishop end would represent a trivial homology class, but its image under the homology class is not, giving a contradiction.

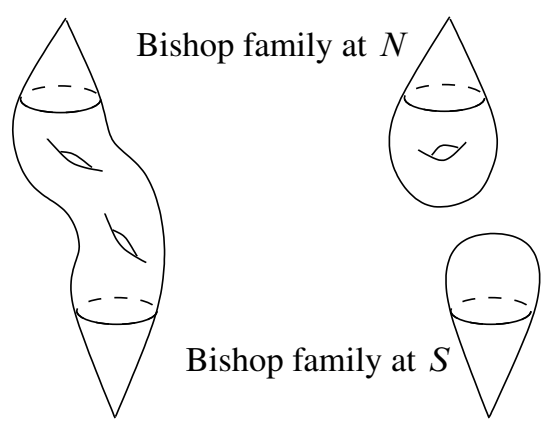

Figure 9: The moduli space has either a single component with two ends or two components each with a single end.

\section{Appendix A Outlook and open problems}

The notion of overtwistedness plays a very central role in 3-dimensional contact topology. The main implications are, as mentioned in the introduction, the nonfillability and the easy classification of such manifolds. We have tried to generalize the definition to higher dimensions, and have proved that our definition implies nonfillability, but clearly, the easy classification of overtwisted 3-manifolds is for contact topology the 
more important of the two properties. In particular, it has been shown that any oriented 3-manifold supports overtwisted contact structures. Thus it is very disappointing that we have not been able to find a single closed contact manifold of higher dimensions containing a plastikstufe.

An interesting future research goal could consist in trying to find a relation between the plastikstufe and the work of Giroux. He discovered that contact manifolds can be decomposed into open books, which are compatible with the contact structure. He also recognized that any contact open book, whose monodromy is composed only by right-handed Dehn twists, leads to a fillable contact manifold. Guided by this realization, one could try to find for example a plastikstufe in the sphere $\left(\mathbb{S}^{2 n+1}, \alpha_{-}\right)$, whose open book decomposition has page $P \cong\left(T^{*} \mathbb{S}^{n}, d \lambda_{\text {can }}\right)$ and whose monodromy consists of a single left-handed Dehn twist. In the 3-dimensional case, it is easy to find explicitly an overtwisted disk. More generally, if a plastikstufe could be found, one could ask:

Question 1 Can one read off from an open book decomposition, whether the contact manifold, contains a plastikstufe?

Finding examples of closed manifolds is the most immediate problem, but other questions are also interesting. The only application of the plastikstufe so far is the detection of exotic contact structures on $\mathbb{R}^{2 n-1}$. There are many constructions leading to such manifolds, which could potentially lead to nonequivalent contact forms. Use for example different overtwisted contact structures on $\mathbb{R}^{3}$ in Section 5.1 to create exotic structures on $\mathbb{R}^{7}$.

Question 2 Can one somehow distinguish some of the exotic contact structures on $\mathbb{R}^{2 n-1}$ containing a plastikstufe?

In dimension 7 and higher, there are many different plastikstufes, because one can use different choices for the singular set $S$.

Question 3 Are the different versions of the plastikstufe in higher dimension somehow equivalent?

\section{Appendix B The almost complex structure on the cotangent bundle}

The following statements about the cotangent bundle can certainly be found in many references, but for completeness, we still repeat them here: the aim will be to associate 
to any Riemannian manifold $(M, g)$ a natural metric on the cotangent bundle $T^{*} M$ (ie a bundle metric on $T\left(T^{*} M\right)$ ), and use this to choose an almost complex structure $J_{g}$ on $T^{*} M$.

Let $\pi: T^{*} M \rightarrow M$ be the standard projection, and let $g^{\dagger}$ be the bundle metric on $T^{*} M$ induced by $g$. The vertical bundle $V\left(T^{*} M\right)$ of $T\left(T^{*} M\right)$, ie the kernel of $\pi_{*}: T\left(T^{*} M\right) \rightarrow T M$, can be naturally identified with the bundle $\pi^{*}\left(T^{*} M\right)$ over $T^{*} M$ by taking two covectors $\beta_{1}, \beta_{2} \in T_{p}^{*} M$, and considering the derivative of the path $\beta_{1}+t \beta_{2} \subset T_{p}^{*} M$ at $t=0$. This identification makes it possible to use $g^{\dagger}$ to define a bundle metric on $V\left(T^{*} M\right)$.

The Levi-Civita connection gives the natural splitting

$$
T\left(T^{*} M\right)=H\left(T^{*} M\right) \oplus V\left(T^{*} M\right)
$$

into horizontal and vertical bundle. Denote the vertical part of a vector $v \in T\left(T^{*} M\right)$ by $v_{V}$ and the horizontal one by $v_{H}$. This splitting induces a natural metric $\tilde{g}$ on $T^{*} M$ (ie a bundle metric on $T\left(T^{*} M\right)$ )

$$
\widetilde{g}(v, w):=g\left(\pi_{*} v, \pi_{*} w\right)+g^{\dagger}\left(v_{V}, w_{V}\right),
$$

where we used the natural identification of the vertical bundle described above.

Theorem 13 There is a unique almost complex structure $J_{g}$ on $T^{*} M$ that is compatible with $d \lambda_{\text {can }}$ and $\tilde{g}$. Furthermore the function

$$
F(\mathbf{q}, \mathbf{p}):=\frac{1}{2} g^{\dagger}(\mathbf{p}, \mathbf{p})
$$

on $T^{*} M$ satisfies

$$
d F \circ J_{g}=-\lambda_{\text {can }} .
$$

Proof We need to check that there is a unique solution $J_{g}$ for the equation

$$
d \lambda_{\text {can }}\left(\cdot, J_{g} \cdot\right)=\tilde{g}(\cdot, \cdot),
$$

such that $d \lambda_{\text {can }}\left(J_{g} \cdot, J_{g} \cdot\right)=d \lambda_{\operatorname{can}}(\cdot, \cdot)$ and $J_{g}^{2}=-\mathbb{1}$.

The equations are independent of any chart, hence it suffices to check them at every point in one special chart explicitly. Choose for a point $\mathbf{q}_{0} \in M$ a geodesic normal chart, ie coordinates $\left(q^{1}, \ldots, q^{n}\right)$ such that the $\left\langle\partial_{q^{1}}, \ldots, \partial_{q^{n}}\right\rangle$ form at $\mathbf{q}_{0}$ an orthonormal basis, and such that all Christoffel symbols vanish at that point. For the cotangent bundle, we obtain a chart $\left(q^{1}, \ldots, q^{n}, p_{1}, \ldots, p_{n}\right)$, where the vertical bundle

$$
V_{\left(\mathbf{q}_{0}, \mathbf{p}\right)}\left(T^{*} M\right)=\left\{\left(\mathbf{q}_{0}, \mathbf{p} ; 0, \dot{\mathbf{p}}\right) \mid \mathbf{p}, \dot{\mathbf{p}} \in \mathbb{R}^{n}\right\},
$$


and horizontal bundle

$$
H_{\left(\mathbf{q}_{0}, \mathbf{p}\right)}\left(T^{*} M\right)=\left\{\left(\mathbf{q}_{0}, \mathbf{p} ; \dot{\mathbf{q}}, 0\right) \mid \mathbf{p}, \dot{\mathbf{q}} \in \mathbb{R}^{n}\right\}
$$

at $T_{\mathbf{q}_{0}}^{*} M$ take the very easy form written above. The metric $\tilde{g}$ can be represented at $\mathbf{q}_{0}$ in the chart by the matrix $\left(\begin{array}{ll}\mathbb{1} & 0 \\ 0 & \mathbb{1}\end{array}\right)$, and the 2 -form $d \lambda_{\text {can }}$ by $\left(\begin{array}{cc}0 & \mathbb{1} \\ -1 & 0\end{array}\right)$. It follows that at the given point, $J_{g}$ is the map that sends $\partial_{q^{j}}$ to $\partial_{p_{j}}$ and $\partial_{p_{j}}$ to $-\partial_{q^{j}}$. This solves the first claim of the theorem.

To check the equality for the function $F$, we will again use a normal geodesic chart around $\mathbf{q}_{0}$ as explained above. A short computation at $\mathbf{q}_{0}$ shows that (the $g^{i j}$ denote the coefficients of the metric $g^{\dagger}$ )

$$
d F=\frac{1}{2} \frac{\partial g^{i j}}{\partial q^{k}} p_{i} p_{j} d q^{k}+g^{i j} p_{i} d p_{j}=p_{j} d p_{j} .
$$

Since $J_{g}$ sends $\partial_{q^{j}}$ to $\partial_{p_{j}}$, it follows that

$$
d F \circ J_{g}=p_{j} d p_{j} \circ J_{g}=p_{j} d q^{j}=-\lambda_{\text {can }}
$$

at the considered point, but since there is a geodesic normal chart around any point, the equation holds everywhere.

\section{References}

[1] L Bates, G Peschke, A remarkable symplectic structure, J. Differential Geom. 32 (1990) 533-538 MR1072917

[2] T Ekholm, J Etnyre, M Sullivan, The contact homology of Legendrian submanifolds in $\mathbb{R}^{2 n+1}$, J. Differential Geom. 71 (2005) 177-305 MR2197142

[3] Y Eliashberg, Classification of overtwisted contact structures on 3-manifolds, Invent. Math. 98 (1989) 623-637 MR1022310

[4] Y Eliashberg, Filling by holomorphic discs and its applications, from: "Geometry of low-dimensional manifolds, 2 (Durham, 1989)", London Math. Soc. Lecture Note Ser. 151, Cambridge Univ. Press, Cambridge (1990) 45-67 MR1171908

[5] H Geiges, Contact geometry, from: "Handbook of differential geometry. Vol. II", Elsevier/North-Holland, Amsterdam (2006) 315-382 MR2194671

[6] D Gilbarg, NS Trudinger, Elliptic partial differential equations of second order, Classics in Mathematics, Springer, Berlin (2001) MR1814364Reprint of the 1998 edition

[7] E Giroux, Géométrie de contact: de la dimension trois vers les dimensions supérieures, from: "Proceedings of the International Congress of Mathematicians, Vol. II (Beijing, 2002)”, Higher Ed. Press, Beijing (2002) 405-414 MR1957051 
[8] M Gromov, Pseudoholomorphic curves in symplectic manifolds, Invent. Math. 82 (1985) 307-347 MR809718

[9] D McDuff, D Salamon, Introduction to symplectic topology, second edition, Oxford Mathematical Monographs, The Clarendon Press Oxford University Press, New York (1998) MR1698616

[10] D McDuff, D Salamon, J-holomorphic curves and symplectic topology, American Mathematical Society Colloquium Publications 52, American Mathematical Society, Providence, RI (2004) MR2045629

[11] M-P Muller, Une structure symplectique sur $\mathbf{R}^{6}$ avec une sphère lagrangienne plongée et un champ de Liouville complet, Comment. Math. Helv. 65 (1990) 623663 MR1078102

[12] F Presas, A class of non-fillable contact structures arXiv:math.SG/0611390

Département de Mathématiques, Université Libre de Bruxelles, CP 218

Boulevard du Triomphe, B-1050 Bruxelles, Belgium

kniederk@ulb.ac.be

Received: 11 September 2006 Revised: 17 November 2006 